\title{
COMPLEX MANIFOLDS AND MATHEMATICAL PHYSICS
}

\author{
BY R. O. WELLS, JR. ${ }^{1}$
}

\section{TABLE OF CONTENTS}

1. Introduction

2. The interaction of complex manifold theory with mathematical physics: A summary

3. Minkowski space

4. Twistors and the Penrose correspondence

5. Homogeneous spaces and group actions

6. Tensors and spinors

7. Maxwell's equations and the zero-rest-mass field equations

8. Cohomology and holomorphic vector bundles

9. Holomorphic representation of solutions of the zero-rest-mass field equations

10. Deriving the zero-rest-mass field equations from integral geometry.

1. Introduction. In the past several years there have been some remarkable links forged between two rather distinct areas of research, namely complex manifold theory on the one hand, and mathematical physics on the other. Complex manifold theory has its roots in the theory of Riemann surfaces and in algebraic geometry, and has seen significant progress in this century based on the introduction of ideas from algebraic topology, differential geometry, partial differential equations, etc. Mathematical physics has been involved in this century in the developments of relativity theory, quantum mechanics, quantum electrodynamics, and quantum field theory, to mention some major developments. Most of these disciplines are formulated in forms of field equations, i.e. partial differential equations whose solutions (under some boundary conditions) represent physical or measurable quantities. The link mentioned above between complex manifold theory and mathematical physics is that in many cases, the solutions of a given field equation can be represented entirely in terms of complex manifolds, holomorphic vector bundles, or cohomology classes on open complex manifolds with coefficients in certain holomorphic vector bundles. In simplistic terms the field equations can be reduced to the Cauchy-Riemann equations by making suitable changes in the geometric background space.

The purpose of this paper is to survey some of these interactions which

Received by the editors July 1, 1978.

AMS (MOS) subject classifications (1970). Primary 32-02, 32C35, 32L05, 32L10, 32G05, 53C65, $83 \mathrm{C} 50$.

${ }^{1}$ The preparation of this paper was supported by NSF MCS 75-05270 at Rice University, by the University of Paris VI, and by Oxford University. 
have been under intense investigation during the past several years.

In $\$ 2$ we give a survey of some of the principal interactions of complex manifold theory and mathematical physics that we are familiar with. In the remainder of the paper, we pick on one of the themes mentioned in $\$ 2$ and develop it in more detail. Namely, we study the representation of the solution of Maxwell's equations (and more generally, but with no more work, the zero-rest-mass field equations) in terms of cohomology classes on certain open subsets of $\mathbf{P}_{3}(\mathbf{C})$ with coefficients in certain holomorphic line bundles.

In $\$ 3$ we review the geometry of Minkowski space. In $\$ 4$ we introduce the Penrose correspondence between the space of twistors and Minkowski space. This is the "change in background space" referred to above, in which points in space-time become complex projective lines in $\mathbf{P}_{\mathbf{3}}$ (= projective twistors), and points in a specific real hypersurface in $\mathbf{P}_{3}$ become null lines or light rays in Minkowski space. The geometry of twistor space is very important, and it is studied in some detail in $\$ \$ 4$ and 5 . In $\$ 6$ we introduce the language of spinors, which enables us to write down certain equations of mathematical physics in a compact form, and this is carried out in \$7. In \$8 we survey briefly the basic concepts of holomorphic vector bundles and cohomology on complex manifolds. $\$ 9$ is devoted to showing how certain cohomology classes, via the Penrose correspondence, yield solutions of these zero-rest-mass field equations (including Maxwell's equations). In $\$ 10$ we present briefly an account of why the zero-rest-mass field equations arise naturally from the Penrose correspondence.

I would like to express my gratitude to Roger Penrose, whose lectures at Pittsburgh in the summer of 1976 inspired me to learn more about this subject, for his hospitality and long discussions at Oxford University, and for commenting on the first draft of this paper. Also, I'd like to thank P. Dolbeault, P. Lelong, and P. Malliavin for their invitation to lecture at the University of Paris VI, where these notes were first written. Finally, I'd like to thank Isadore Singer and Richard Ward who gave me helpful comments on my first draft; in particular Singer suggested to me that the zero-rest-mass field equations should be a consequence of the "integral geometry" of the Penrose correspondence, which I have described briefly in $\$ 10$.

2. The interaction of complex manifold theory with mathematical physics: $A$ summary. We want to give a brief survey of some of the recent interactions between certain areas of mathematical physics and the general theory of complex manifolds. This includes, in particular, the relativistic wave equations for particles of zero-rest-mass and Einstein's equations of general relativity. Most of the interactions depend on a correspondence between $\mathbf{P}_{3}(C)$ and a complexification of compactified Minkowski space due to Roger Penrose. This correspondence has the property of transferring problems in mathematical physics in Minkowski space into problems of several complex variables on (subsets of) $\mathbf{P}_{\mathbf{3}}(\mathbf{C})$. There are various levels of interactions and we will give a somewhat historical survey.

The topics in physics we will attempt to describe in terms of holomorphic objects include:

(1) Minkowski space $M_{0}=\left\{\mathbf{R}^{4}\right.$ equipped with a flat Lorentz metric of signature $(+,-,-,-)\}(\$ 3)$. 
(2) Solutions of Maxwell's equations in $M_{0}(\$ 7)$.

(3) Solutions of the Zero-Rest-Mass Field Equations of spin $s$ in $M_{0}$ :

$s=\frac{1}{2}:$ Dirac-Weyl equation of a neutrino,

$s=1:$ Maxwell's equations,

$s=2$ : Linearized Einstein's gravitational equations corresponding to "weak gravitational fields" (see §7).

(4) Solutions of Einstein's gravitational field equations for curved spacetime.

(5) Solutions of the Yang-Mills field equations for arbitrary gauge groups.

The first three topics are discussed in further detail in later sections of the paper. The latter two topics are discussed briefly in this introduction.

All of these equations can be described in various explicit forms using certain notations which the mathematical physicists have developed over the years, including special coordinate systems, tensors, van der Waerden's 2spinor notation, some of which will be developed later in this paper. In this section we will suppress the notation of mathematical physics and discuss the physical fields of interest in general terms to get an idea of what kind of holomorphic objects are useful in their representations.

A. TWISTOR GEOMETRY. This is the basis for the applications of complex manifold theory for mathematical physics. Briefly,

$$
\mathbf{T}=\{\text { twistors }\}=\left\{\mathbf{C}^{4} \text { with an Hermitian form } \Phi \text { of signature }(++--)\right\} \text {. }
$$

Let

$$
\begin{aligned}
\mathbf{T}^{+} & =\{Z \in \mathbf{T}: \Phi(Z)>0\} \text {-positive twistors, } \\
\mathbf{T}^{0} & =\{Z \in \mathbf{T}: \Phi(Z)=0\} \text {-null twistors, } \\
\mathbf{T}^{-} & =\{Z \in \mathbf{T}: \Phi(Z)<0\} \text {-negative twistors. }
\end{aligned}
$$

By going to the projective space we have the corresponding portions of projectivized twistor space $\mathbf{P}(\mathbf{T})=\mathbf{P}_{3}(\mathbf{C})$ and $\mathbf{P}_{3}^{+}, \mathbf{P}_{3}^{0}$, and $\mathbf{P}_{3}^{-}$(having homogeneous coordinates in $\mathbf{T}^{+}, \mathbf{T}^{0}, \mathbf{T}^{-}$respectively). We let $N=\mathbf{P}_{3}^{0}$, and we see that $N$ is a real 5-dimensional hypersurface in $\mathbf{P}(\mathbf{T})$ which divides $\mathbf{P}(\mathbf{T})$ into the two complex-analytically equivalent parts, $\mathbf{P}_{3}^{+}$and $\mathbf{P}_{3}^{-} \cdot \mathbf{P}_{3}^{+}$and $\mathbf{P}_{3}^{-}$are in particular not Stein manifolds, and admit no nonconstant holomorphic functions. The complex manifolds $\mathbf{P}_{3}^{+}, \mathbf{P}_{3}^{-}$and their common topological boundary $N$, which is a real-analytic hypersurface (with Levi form having two eigenvalues of opposite sign), is where the holomorphic objects of interest will have their domain of definition. The space of twistors is a representation space for $S U(2,2)$ which is a 4-1 covering of the conformal group acting on (compactified) Minkowski space which will be discussed in §3. This is analogous to spinors which are a representation space for $\operatorname{SL}(2, \mathrm{C})$, a 2-1 covering of the Lorentz group. The conformal group contains the Lorentz group as a proper subgroup (cf. §3), so twistors are generalizations of spinors. The field equations of particles which move at the speed of light (zero-restmass), are conformally invariant, and thus amenable to study in terms of twistors (neutrinos, photons; etc.). Particles with nonzero rest mass have also been studied recently in terms of twistors, but that is less well understood (Hughston [13], Penrose [23]). 
Let $M$ be compactified Minkowski space, which is a compact 4dimensional Lorentzian manifold $\left(\cong S^{1} \times S^{3}\right)$ which has an open dense subset conformally equivalent to flat Minkowski space $M_{0}$ (cf. Kuiper [13], Penrose [17]). Heuristically, $M=M_{0} \cup$ \{light cone at $\infty$ \}. It can be explicitly realized as a quadric in $\mathbf{P}_{5}(\mathbf{R})$, where the conformal group is induced by projective transformations.

There is a Penrose correspondence between $M$ and $N \subset \mathbf{P}(\mathrm{T})$ of the form

$$
\begin{gathered}
\{\text { complex lines in } N\} \underset{\text { correspondence }}{\stackrel{1-1}{\leftrightarrow}}\{\text { points in } M\}, \\
\{\text { points in } N\} \underset{\text { correspondence }}{\stackrel{1-1}{\leftrightarrow}}\{\text { null lines in } M\} .
\end{gathered}
$$

Null lines are curves whose tangent vectors are null with respect to the Minkowski metric, and it's clear that the set of null lines depends only on the conformal structure of $M$, not on any specific choice of metric. These are the paths of motion of zero-rest-mass particles. More generally we can introduce a complex manifold $M_{\mathbf{C}}$ of 4 complex dimensions which is a complexification of $M$ so that the correspondence above extends:

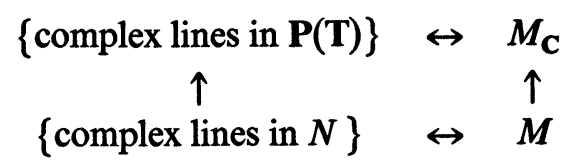

and $M_{\mathrm{C}}$ turns out to be nothing other than the Grassmanian manifold of 2-dimensional complex planes in $\mathbf{C}^{4}, G_{2,4}(\mathbf{C})$, which is clearly equivalent to the set of all complex lines in $\mathbf{P}_{3}(\mathbf{C})$. This correspondence was developed with many applications in [17]-[23]. It will be studied in more detail in $\$ 4$.

B. ROBINSON-KERR THEOREM. Consider a null solution of the homogeneous Maxwell's equation in $M_{0}$, i.e. a 2-form $F$ on $M_{0}$ satisfying:

(1) $d F=d^{*} F=0$ (Maxwell),

(2) $\|F\|=\|* F\|=0$ (nullity),

where * is the Hodge duality operator and $d^{*}$ is the adjoint of $d$ with respect to the Lorentz metric on $M_{0}$. These are a special type of "symmetric" solutions to Maxwell's equation where the electric and magnetic fields have equal intensities and are orthogonal. Robinson [26] showed that there is a correspondence between (local) null solutions of Maxwell's equations and shear-free null congruences ( $=$ shear-free null 1-dimensional foliations). Here shear-free corresponds to a first order differential equation satisfied by the vector field generating the congruence (or foliation).

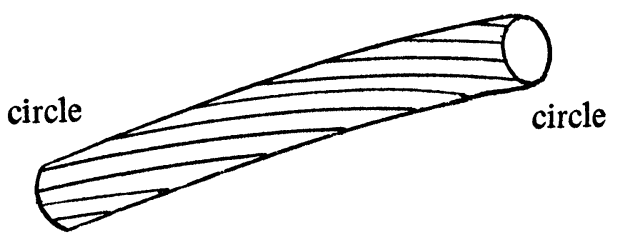

twisting, but shear-free flow lines

FIGURE 2.1 
This corresponds to the usual notion of "shear" in classical continuum mechanics. For our purposes here we won't make that notion precise, but the flow lines correspond to the flow of electromagnetic radiation, and it cannot flow in an arbitrary manner, but is restricted by the shear-free condition. Now any null congruence is locally a 3-dimensional family of light rays (null lines), so by the Penrose correspondence in $\$ 2 \mathrm{~A}$ we see that this corresponds to a 3-dimensional set of points in $N \subset \mathbf{P}(\mathbf{T})$. Let the congruence be denoted by $C$, and let the corresponding parametrizing submanifold in $N$ be denoted by $\tilde{C}$, then we have the Kerr theorem: If the congruence is real-analytic, then

$$
C \text { is shear free } \Leftrightarrow \tilde{C}=N \cap V_{\mathbf{C}} \text {, }
$$

where $V_{C}$ is a locally defined complex-analytic submanifold of 2-complex dimensions.

This is developed in Penrose [17], and there is a new proof in [9].

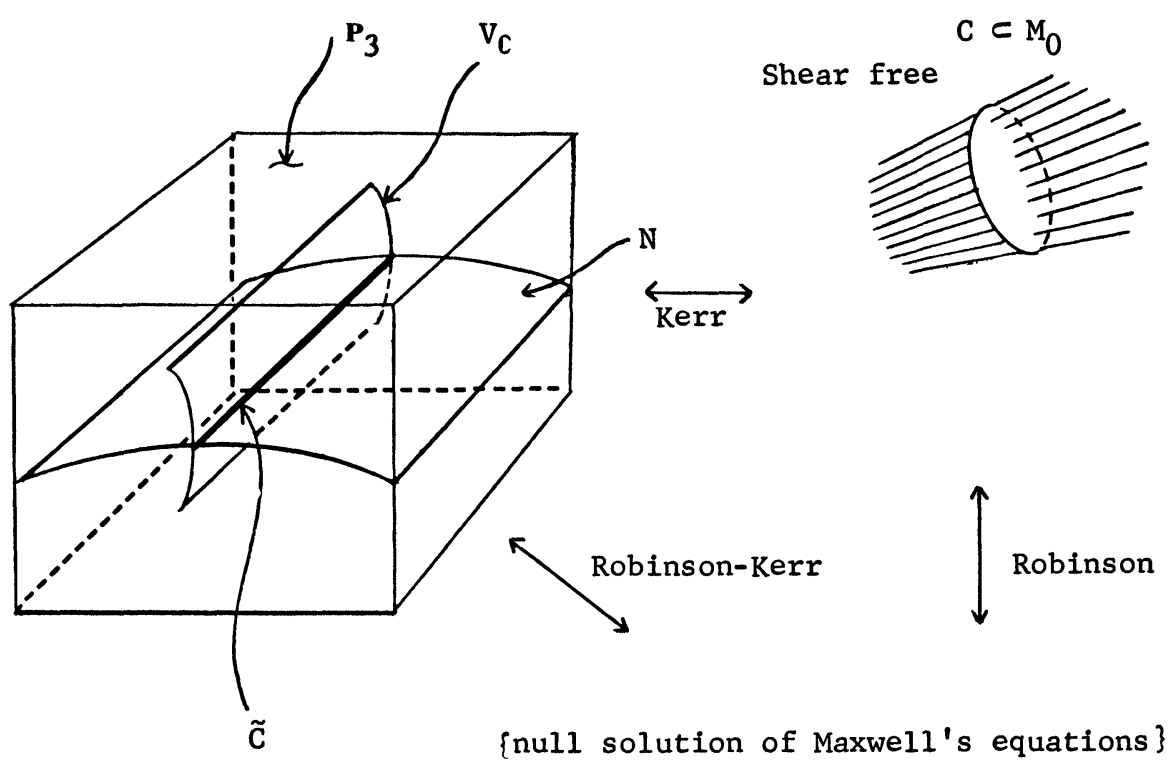

FIGURE 2.2

This was the first time complex analysis objects (the complex submanifold $V_{\mathrm{C}}$ ) entered into the study of field equations in the twistor context and was the motivation for most of the later work. This result has been generalized by R. Penrose and D. Lerner (cf. [14]), who noted that if $C$ were only of class $C^{1}$ then $\tilde{C}$ is maximally complex in $\mathbf{P}_{3}$ (i.e. in this case, its holomorphic tangent space at each point has complex dimension 1). There are still many unresolved global questions concerning this result, in particular, its relationship to the recent work of Harvey-Lawson [10].

C. Contour InTEgrals. The Robinson-Kerr Theorem was generalized by Penrose's contour integral representation of solutions of Maxwell's equations, which no longer had to be null. Let $\left(z_{1}, z_{2}, z_{3}\right)$ be local coordinates in $\mathbf{P}(\mathbf{T})$ 
and consider an affine mapping

$$
\begin{aligned}
M_{0} \times \mathbf{C} & \stackrel{x}{\rightarrow} \mathbf{P}(\mathbf{T}), \\
(m, \lambda) & \mapsto\left(z_{1}, z_{2}, z_{3}\right) .
\end{aligned}
$$

Define

$$
\varphi_{r}(m)=\frac{1}{2 \pi i} \oint_{K_{m}} \lambda^{r} f(\chi(\lambda, m)) d \lambda, \quad r=0,1,2,
$$

where $f$ is holomorphic in $\left(z_{1}, z_{2}, z_{3}\right)$ except at some singular points, $K_{m}$ is a smoothly varying contour in $\chi(\{m\} \times \mathbf{C})$ (a complex line in $\mathbf{P}_{3}$ ), and $f$ is holomorphic on the contour $K_{m}$. For a suitable choice of $\chi$ one obtains that if $f$ is homogeneous of degree -4 in $\left(z_{1}, z_{2}, z_{3}\right)$ then $\varphi_{0}, \varphi_{1}, \varphi_{2}$ determine local solutions to Maxwell's equations. Namely, if

$$
F=F^{+}+F^{-}
$$

where $* F^{+}=i F^{+}, * F^{-}=-i F^{-}$, then

$$
F^{-}=F_{a b}^{-} d x^{a} \wedge d x^{b}
$$

corresponds to (in spinor notation, see §6),

$$
\phi_{A B} \varepsilon_{A^{\prime} B^{\prime}}
$$

where $\varphi_{00}=\varphi_{0}, \varphi_{10}=\varphi_{1}, \varphi_{11}=\varphi_{2}, \varphi_{A B}$ is symmetric in $A, B, \varepsilon_{A^{\prime} B^{\prime}}$ is a constant skewsymmetric matrix (see §7). Basically $\varphi_{0}, \varphi_{1}$, and $\varphi_{2}$ given by the above contour integral formula determine $F^{-}$by algebraic means, and a similar representation is possible for $F^{+}$. Thus we say that solutions of Maxwell's equations are obtainable by means of contour integrals. This was developed in [19], [20], and extensive expositions of this is given in [25] and [21] with many examples worked out in detail.

REMARKS. (1) All real-analytic solutions are so obtainable.

(2) If $f$ has no pole or singularity inside the contour then we get the zero solution.

(3) If $f$ has a simple pole inside the contour, then we get null solutions, and this is compatible with the "Robinson-Kerr solution" above. The divisor defined by the simple pole gives a shear-free congruence which corresponds to a null solution to Maxwell's equation, by the Robinson-Kerr theorem.

(4) This gives only real-analytic solutions, but if we extend to the complex domain in $\boldsymbol{M}_{\mathbf{C}}$, then we'll obtain holomorphic solutions, and general nonreal-analytic solutions can be represented in terms of boundary values of holomorphic function, e.g., $C^{\infty}$ distributions, or hyperfunction solutions.

(5) The same procedure works for zero-rest-mass fields of spin $s \geqslant 0$, by requiring $f$ to be homogeneous of degree $-2 s-2$ (see $\$ 7$ ).

D. Cономоlogy Classes. The above description of solutions of Maxwell's equations is not Poincare or conformally invariant and the function $f$ was not determined uniquely by the field. This has been improved by recognizing that the function $f$ which was used above is a local representation of a cohomology class with coefficients in a certain bundle depending on the homogeneity. 


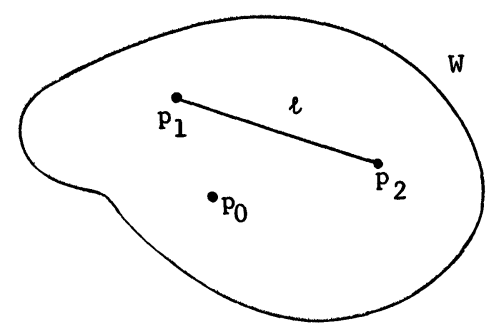

FIGURE 2.3

This led to the correspondence (cf. Penrose [23])

$$
\begin{aligned}
& H^{0,1}\left(\overline{\mathbf{P}_{3}^{+}}, H^{-2 s-2}\right) \leftrightarrow \quad \text { \{wave functions of } \\
& \text { helicity } s \text { for } \\
& \text { a massless particle\} }
\end{aligned}
$$

where $H \rightarrow \mathbf{P}_{3}$ is the hyperplane section bundle of $\mathbf{P}_{3}$. This is described in more detail in $\$ \S 8-10$.

The correspondence above between cohomology and wave functions can be carried out explicitly by one of several techniques:

(a) branched contour integrals (cf. [25]).

(b) using a Dolbeault representation, $\varphi=\int_{\mathbf{P}_{1}} \ldots \alpha \wedge \theta, \alpha$ a $\bar{\partial}$-closed $(0,1)$-form representing a class in $H^{0,1}\left(\overline{\mathbf{P}_{3}^{+}}, H^{-2 s-2}\right)$, and $\theta$ a specific $(1,0)$ form ([35], see §8),

(c) Ward-Sparling "splitting method" [29].

This correspondence can be realized formally and invariantly from the point of view of integral geometry (cf. §10).

E. EINSTEIN'S GRAVITATIONAL EQUATIONS. One of the problems of general relativity is to find the most general solution to Einstein's equations. Basically Einstein's vacuum equation asserts that a 4-dimensional Lorentz manifold should have Ricci curvature $=0$. This is a nonlinear differential equation for the components of the metric tensor. A smooth perturbation of flat Minkowski space which is still Lorentzian, and with nontrivial curvature will not necessarily satisfy Einstein's equations. Using the Penrose correspondence between Minkowski space and projective twistor space, Penrose uses deformation of the complex structure of open subsets of $\mathbf{P}_{3}$ to generate the most general self-dual holomorphic solutions to Einstein's equations. It's not clear at present how to get rid of the self-dual restriction (which arises naturally in this context) in order to obtain the most general holomorphic solution, or how to obtain real solutions from these complex ones. It is however, a large class of nontrivial solutions generated in a systematic manner, as opposed to many of the special solutions obtained over the last 60 years.

The idea is, very briefly, as follows. Let $p_{0}$ be a point in $M_{\mathrm{C}}$, and $W$ a neighborhood of $p_{0}$. The point $p_{0}$ corresponds, under the Penrose correspondence, to a complex line $L_{0} \subset \mathbf{P}_{3}$. The neighborhood $W$ corresponds to a neighborhood $U$ of $L_{0}$. Let $p_{1}$ and $p_{2}$ be two points in $W$ which are connected by a null line in $W$, then $p_{1}$ and $p_{2}$ correspond to complex lines $L_{1}$ and $L_{2}$ which intersect in $U$ (the point of intersection $Q$ corresponding to the null line $l$ in $W$, cf. Figures 2.3 and 2.4). 


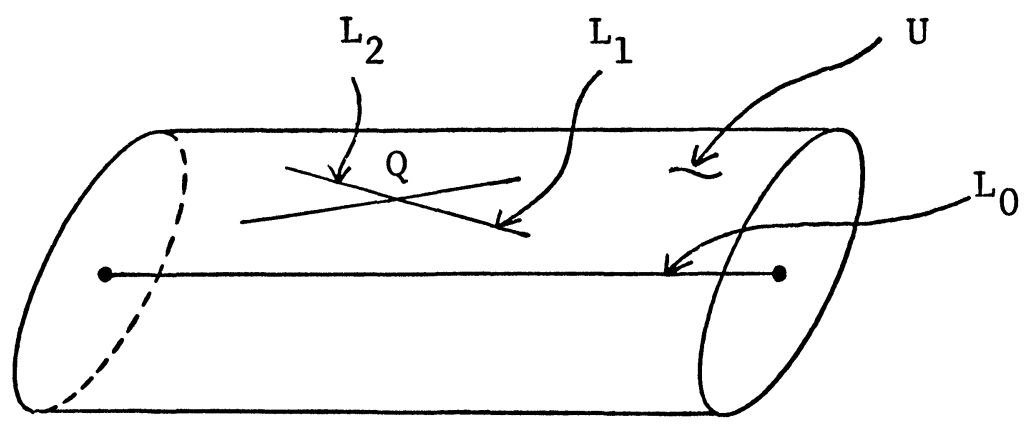

FIGURE 2.4

One knows that, in fact $L_{1}$ intersects $L_{2}$ if and only if $p_{1}$ and $p_{2}$ are connected by a null line in $M_{\mathbf{C}}$ (part of the basic twistor geometry). Now deform the complex structure of $U$ in an arbitrary manner (which one can do explicitly in examples), getting a "nonlinear" $\tilde{U}$ pictured schematically in Figure 2.5.

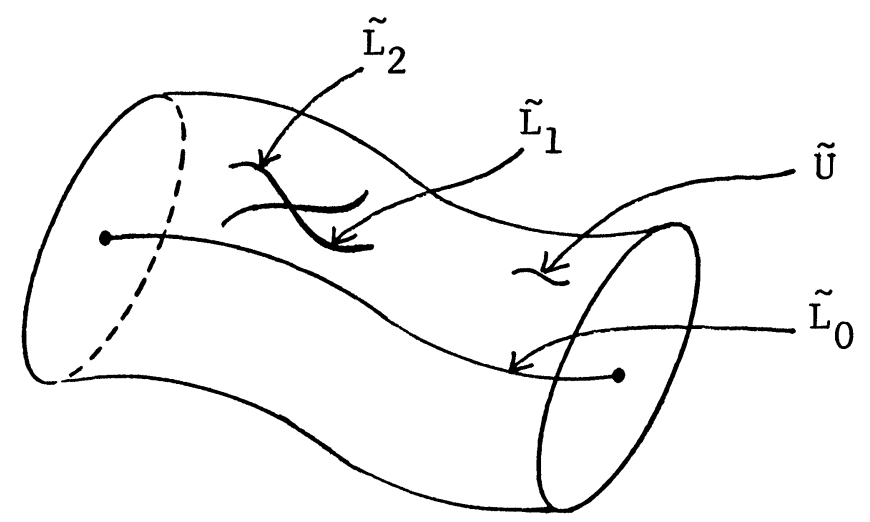

FIGURE 2.5

A theorem of Kodaira tells us that there is in the deformation $\tilde{U}$ a 4-dimensional complex-analytic family of holomorphic curves homologous to the 4-dimensional family of complex lines in $U$. Define $\tilde{W}$ to be the parameter space for this family of holomorphic curves, which is a 4-dimensional complex manifold. Then $\tilde{W}$ is a neighborhood of some fixed point $\tilde{p}_{0}$ corresponding to a fixed holomorphic curve $\tilde{L}_{0}$. If $\tilde{p}_{1}$ and $\tilde{p}_{2}$ are two points in $\tilde{W}$, then we will define $\tilde{p}_{1}$ and $\tilde{p}_{2}$ to be null-separated if and only if the corresponding curves $\tilde{L}_{1}$ and $\tilde{L}_{2}$ intersect. This defines a general self-dual conformal structure on $\tilde{W}$. By additional choices, one can define in this conformal class, a holomorphic Lorentz metric on $\tilde{W}$ which satisfies Einstein's vacuum equations (generically with nontrivial curvature). Further details on this construction are in Penrose [22].

F. YANG-Mills FIELD EQUATIONS. Maxwell's equations describe electromagnetic interactions. The Yang-Mills field equations describe a class of interactions of particles called weak interactions which occur, for instance, 
in certain kinds of radioactive decay. The Yang-Mills field equations are a nonlinear generalization of Maxwell's equations arising in gauge theory. They can be described in terms of connections and curvature on vector bundles on space-time. (Cf. Atiyah-Hitchen-Singer [2].) It has been of interest for some time to physicists to obtain information about solutions to these equations.

Recently Ward showed how solutions of Maxwell's equations correspond to holomorphic line bundles over open subsets of $\mathbf{P}_{3}$ by using the Penrose correspondence (the "twisted photon," [28]), and he generalized this construction to show how solutions of the Yang-Mills equation corresponds to holomorphic vector bundles of rank 2 over open subsets of $\mathbf{P}_{3}$ (Ward [30], [31]). Atiyah and Ward [3], and also Atiyah-Hitchen-Singer [2] have used this correspondence to obtain various kinds of information about solutions of the Yang-Mills field equations as a consequence of numerous recent results concerning the classification of holomorphic vector bundles of rank 2 over $\mathbf{P}_{3}$ (e.g. Barth [4]). We won't go into these matters in any further detail in this paper, but refer to the literature cited above.

ADDED IN PROOF. There has been notable progress in the understanding of the solutions to the Yang-Mills equations in an important special case. In a recent paper (M. F. Atiyah, N. Hitchen, B. G. Drinfeld, and Yu. I. Manin, Construction of instantons, Physics Letters 65A (1978), 185-187) is described a complete set of solutions to the self-dual Yang-Mills equations in the Euclidean metric on the 4-sphere $S^{4}$ (the "instanton" case). This solution uses the full power of the Ward correspondence of solutions of the equations with vector bundles on $\mathbf{P}_{3}$ and reduces the problem of finding solutions of the self-dual Yang-Mills equations to a straightforward problem in quaternionic linear algebra.

3. Minkowski space. In this section we summarize briefly the geometry of classical Minkowski space. By definition Minkowski space is $\mathbf{R}^{4}$ equipped with a flat Lorentz (pseudo) metric of signature -2 , i.e. in appropriate coordinates the metric tensor is diagonal with diagonal entries $\{+1,-1,-1,-1\}$. We denote Minkowski space by $M_{0}$, and we denote the inner product on the tangent space at $p \in M_{0}$ by $(,)_{p}$, and the norm of a tangent vector by \|\|$_{p}$. A tangent vector $X \in T_{p}\left(M_{0}\right)$ is said to be null if and only if $\|X\|_{p}=0$. The set of null vectors at $p$ is said to be the null cone or light cone at $p$. The Poincaré group $P$ is the group of isometries of $M_{0}$, and the Lorentz group at $p \in M_{0}$ is the subgroup $L_{p}$ of $P$ which leaves $p$ fixed. It's clear that $L_{p} \cong O(1,3)$, and that $P$ is isomorphic to the semidirect product of $O(1,3)$ with the translations in $\mathbf{R}^{4}$. The restricted Lorentz group (suppressing the point $p$ ) $L_{\uparrow}^{+}=O_{\uparrow}^{+}(1,3)$ has determinant +1 and preserves the given space and time orientation ( = connected component of $O(1,3)$ ).

There is no preferred origin to $M_{0}$, and $M_{0}$ is the physicists' basic model of special relativity or flat space-time, where there is no preferred choice of origin or (Lorentzian) frame of reference. If we choose a particular origin $O \in M_{0}$, we can define two classes of transformation at $O$ (which by composition with translations will then be defined at any other point of $M_{0}$ ). 
There are the

$$
\begin{aligned}
& \text { dilations: } x \rightarrow \rho x, \rho \in \mathbf{R}, \\
& \text { inversions: } x \rightarrow-x /\|x\|_{0}^{2} \text {. }
\end{aligned}
$$

Here we've identified $T_{0}\left(\mathbf{R}^{4}\right)$ with $\mathbf{R}^{4}$. The inversions are well defined outside of the null cone at 0 , and both the dilations and the inversions have the property that they preserve the Lorentz metric up to a scale factor, i.e.

$$
\begin{aligned}
(\rho x, \rho x)_{0} & =\rho^{2}\|x\|_{0}^{2}, \\
\left(\frac{-x}{\|x\|_{0}^{2}}, \frac{-x}{\|x\|_{0}^{2}}\right) & =\frac{1}{\|x\|_{0}^{4}}(x, x) .
\end{aligned}
$$

Thus angles are preserved by such mappings, but not length, and they are examples of conformal mappings (with respect to the metric on $M_{0}$ ). The conformal group $C(1,3)$ is the composition of the inversions with the Poincare group $P$. The restricted conformal group $C_{\uparrow}^{+}(1,3)$ is the subgroup which preserves the space and time orientation (the connected component of $C(1,3)$ ). The dilations are generated by $P$ and the inversions automatically, and the inversion (3.1) above does not belong to $C_{\uparrow}^{+}(1,3)$ since it doesn't preserve the space orientation. We have for reference

$$
\operatorname{dim}_{\mathbf{R}} L_{\uparrow}^{+}=6, \operatorname{dim}_{\mathbf{R}} P_{\uparrow}^{+}=10, \operatorname{dim}_{\mathbf{R}} C_{\uparrow}^{+}=15 .
$$

We will see later that the conformal group acts naturally on a compact manifold $M$ which contains $M_{0}$ as a dense open subset, and which we will call the conformal compactification of $M_{0}$. The inversions take the light cone at the origin to a "light cone at $\infty$." Since this compactification will arise naturally in our study of twistors in the next section, we will not give an independent account of the conformal compactification here, but refer to the well-written paper of Kuiper [14] for a discussion of this subject. The compactification turns out to be a real quadric in $\mathbf{P}_{5}(\mathbf{R})$, and the conformal group is represented in terms of projective transformations on $\mathbf{P}_{5}(\mathbf{R})$.

Just as in Riemannian geometry it is useful to work with orthonormal frames, in Minkowski geometry it is convenient to work with an analogous concept, where the basis vectors are null vectors. Let $p \in M_{0}$, and let $X_{0}, X_{1}$, $X_{2}, X_{3}$ be a basis for $T_{p}\left(M_{0}\right)$. If $X=x^{j} X_{j}, x^{j} \in \mathbf{R}$ (summation convention), then $x=\left(x^{0}, x^{1}, x^{2}, x^{3}\right)$ give coordinates for $M_{0}$, and we can choose the basis $\left\{X_{j}\right\}$ such that $(X, Y)_{p}=x^{0} y^{0}-x^{1} y^{1}-x^{2} y^{2}-x^{3} y^{3}$, which we denote also by $(x, y)$. We write $\|x\|=(x, x)^{1 / 2}$. This set of coordinates depends on the choice of the origin at the point $p$, as well as on the particular frame of $\left\{X_{j}\right\}$. There is no preferred choice of origin, but for computational purposes, it's useful to work with this coordinate system, and others related to it.

Let

$$
\begin{array}{ll}
u=\frac{1}{\sqrt{2}}\left(x^{0}+x^{1}\right), & \zeta=\frac{1}{\sqrt{2}}\left(x^{2}+i x^{3}\right), \\
\bar{\zeta}=\frac{1}{\sqrt{2}}\left(x^{2}-i x^{3}\right), & v=\frac{1}{\sqrt{2}}\left(x^{0}-x^{1}\right),
\end{array}
$$

then $(u, v, \zeta, \bar{\zeta})$ is a new set of coordinates in $M_{0}$, and we see easily that $\|u\|=\|v\|=\|\zeta\|=\|\bar{\zeta}\|=0$, where we extend (, ) by complex linearity to 
$M_{0} \otimes_{\mathrm{R}} \mathrm{C}$, the complexification of $M_{0}$. Consider the $2 \times 2$ matrix

$$
m=\left[\begin{array}{ll}
u & \zeta \\
\bar{\zeta} & v
\end{array}\right] .
$$

Then $m$ is Hermitian, and any Hermitian matrix is of this form for some $(u, v, \zeta, \bar{\zeta})$. Moreover,

$$
\operatorname{det} m=u v-\zeta \bar{\zeta}=\frac{1}{2}\|x\|^{2} .
$$

So we see that the norm of a vector $x$ corresponds to the determinant of the $(2 \times 2)$-matrix $m$ in these new coordinates. For many purposes it is convenient to identify $H(2)$, the set of $(2 \times 2)$-Hermitian matrices with the determinant as norm, with Minkowski's space $M_{0}$ with a choice of origin and a choice of basis for $T\left(M_{0}\right)$ at that origin.

The Lorentz mappings at the origin of Minkowski space induce corresponding mappings of the space $H(2)$, and they correspond to:

$$
M_{0} \stackrel{l}{\rightarrow} M_{0} \quad \text { (Lorentz) }
$$

induces

$$
H(2) \stackrel{i}{\rightarrow} H(2)
$$

by $\tilde{l}(m)=s m s^{*}$, where $s \in S L(2, \mathbf{C})$ and $s^{*}=t^{-}$is the Hermitian adjoint. In fact $\operatorname{SL}(2, \mathrm{C})$ is a 2-1 (simply-connected) covering group of $L_{\uparrow}^{+}$, which follows from the above correspondence, noting that $s$ was not uniquely determined by $l$.

4. Twistors and the Penrose correspondence. Our object now is to discuss twistors and see how $M_{0}$ in the preceding section arises in a natural manner in a certain complex-analytic geometric context. Our main tool will be complex flag manifolds, which are natural generalizations of projective spaces and Grassmanian manifolds. They are defined as follows. Consider $\mathbf{C}^{n}$, $n \geqslant 2$, and let $0<d_{1}<\cdots<d_{r}<n$, be integers, and define

$$
\begin{aligned}
F_{d_{1}, \ldots, d_{r}}= & \left\{\left(L_{1}, L_{2}, \ldots, L_{r}\right): L_{1} \subset L_{2} \subset \cdots \subset L_{r} \subset \mathbf{C}^{n}\right. \\
& \text { is a nested sequence of subspaces of } \\
& \left.\mathbf{C}^{n} \text { with } \operatorname{dim}_{\mathbf{C}} L_{j}=d_{j}, j=1, \ldots, r\right\} .
\end{aligned}
$$

The $r$-tuple of subspaces $\left(L_{1}, L_{2}, \ldots, L_{r}\right)$ is called a flag in $\mathbf{C}^{n}$. If $r=1$, $d_{1}=1$, then we see that

$$
F_{1}=\mathbf{P}_{n-1}(\mathbf{C}) \text {, }
$$

the set of complex lines in $\mathbf{C}^{n}$, the usual $(n-1)$-dimensional complex projective space. If $r=1, d_{1}=k$, then $F_{k}=G_{k, n}(\mathrm{C})$, the Grassmanian manifold of $k$-dimensional complex subspaces of $\mathbf{C}^{n}$. It is not difficult to see that $F_{d_{1}, \ldots, d_{r}}$ is a compact complex manifold of complex dimension $d_{1}\left(d_{2}-d_{1}\right)+$ $d_{2}\left(d_{3}-d_{2}\right)+\cdots+d_{r-1}\left(d_{r}-d_{r-1}\right)$, which is moreover homogeneous, with a transitive complex Lie group of automorphisms. Namely the action of $S L(n, \mathbf{C})$ on $\mathbf{C}^{n}$ induces an effective transitive action on $F=F_{d_{1}, \ldots, d_{r}}$ and thus $F=S L(n, \mathrm{C}) / P_{F}$, where $P_{F}$ is the isotropy subgroup at a point of $F$. 
Similarly, $U(n)$ acts transitively, and $F$ is representable as the quotient of compact Lie groups. Using the homogeneous coordinates $\left(z_{1}, \ldots, z_{n}\right) \in \mathbf{C}^{n}$, one can find easily affine coordinate systems for $F$, just as the case for projective space, where one maps $\left(\zeta_{1}, \ldots, \zeta_{n-1}\right) \in \mathbf{C}^{n-1} \rightarrow$ \{subspace spanned by $\left.\left(1, \zeta_{1}, \ldots, \zeta_{n-1}\right)\right\}$ giving affine coordinates for $\mathbf{P}_{n-1}(\mathbf{C})$. For instance, if $z$ is a $2 \times 2$ complex matrix, then the mapping

$$
z \rightarrow\left\{\text { subspace spanned by columns of }\left[\begin{array}{l}
z \\
I_{2}
\end{array}\right]\right\}
$$

gives an affine system of coordinates for $G_{2,4}(\mathbf{C})$, a particular Grassmannian we will be interested in. For further information on complex flag manifolds consult Hirzebruch [11], Wells [32], Wells-Wolf [33] or Wolf [34].

We now want to consider 3 particular complex flag manifolds: From now on we will consider $\mathbf{C}^{4}$ as our basic complex vector space, and with respect to $\mathrm{C}^{4}$ consider the 3 complex flag manifolds $F_{12}, F_{1}$, and $F_{2}$. Then we have the following natural diagram

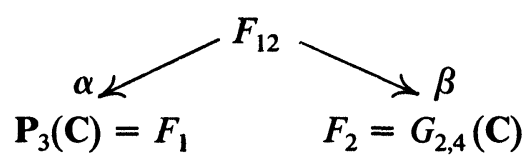

with $\alpha$ and $\beta$ being natural projections, $\alpha\left(L_{1}, L_{2}\right)=L_{1}, \beta\left(L_{1}, L_{2}\right)=L_{2}$. Using $\alpha$ and $\beta$ we can define a correspondence $\tau$ between $F_{1}$ and $F_{2}$, which is a set-valued mapping,

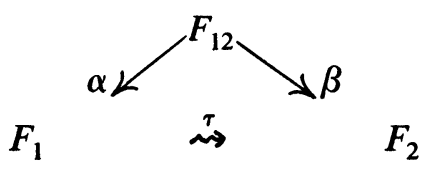

We define $\tau$ by $\tau(p)=\beta\left(\alpha^{-1}(p)\right)$, and $\tau^{-1}(p)=\alpha\left(\beta^{-1}(p)\right)$.

4.2 Proposition. (1) $\tau(p)$ is a 2-complex-dimensional projective plane $\left(\cong \mathbf{P}_{2}(\mathbf{C})\right.$ ) embedded in $F_{2}$.

(2) $\tau^{-1}(p)$ is a 1-complex-dimensional projective line embedded in $F_{1}$.

Proof. (1) By definition,

$$
\alpha^{-1}(p)=\left\{\text { flags }\left(L_{1}^{0}, L_{2}\right): L_{1}^{0} \subset L_{2}, L_{1}^{0} \text {-fixed, } L_{2} \text { variable }\right\}
$$

Therefore

$$
\beta\left(\alpha^{-1}(p)\right)=\left\{L_{2} \subset \mathbf{C}^{4}: L_{2} \supset L_{1}^{0} \text {-fixed }\right\},
$$

i.e. $\beta\left(\alpha^{-1}(p)\right)$ is the set of all 2-dimensional subspaces of $\mathbf{C}^{4}$ which contain a fixed 1-dimensional subspace $L_{1}^{0}$. This is simply an embedding of $\mathbf{P}_{2}(C)$ in $F_{2}$ since, if we fix one vector $e_{1}$, and let $e_{2}$ vary in a 3-dimensional subspace $e_{1}^{\perp}$ perpendicular to $e_{1}$ with respect to some metric on $\mathrm{C}^{4}$, then the span of $\left\{e_{1}, e_{2}\right\}$ will span all subspaces $L_{2} \supset L_{1}^{0}$. But the set of all such $e_{2}$ 's span the set of all complex lines perpendicular to $e_{1}$, which is thus the same as the set of all complex lines in $e_{1}^{\perp}$, and hence is isomorphic to $\mathbf{P}_{2}(\mathbf{C})$.

(2) This is simpler, since by the same reasoning

$$
\alpha\left(\beta^{-1}(p)\right)=\left\{L_{1} \subset \mathbf{C}^{4}: L_{1} \subset L_{2}^{0}, L_{2}^{0} \text {-fixed }\right\} .
$$


But $L_{2}^{0}$ is 2-complex-dimensional, and hence $\alpha\left(\beta^{-1}(p)\right) \cong \mathbf{P}_{1}(\mathbf{C})$. Q.E.D.

We now want to introduce the twistor structure into $\mathbf{C}^{4}$, and see how this affects the correspondence $\tau$ above. Let $\Phi$ be a given nondegenerate Hermitian bilinear form on $\mathbf{C}^{4}$ of signature 0 (i.e. $\{+,+,-,-\}$ ). In appropriate coordinate systems the matrix for $\Phi$ can be represented as

$$
\begin{aligned}
\Phi_{0} & =\left[\begin{array}{cc}
I_{2} & 0 \\
0 & -I_{2}
\end{array}\right], \\
\Phi_{1} & =\left[\begin{array}{cc}
0 & i I_{2} \\
-i I_{2} & 0
\end{array}\right], \\
\Phi_{2} & =\left[\begin{array}{cc}
0 & I_{2} \\
I_{2} & 0
\end{array}\right] .
\end{aligned}
$$

It is easy to check that the Hermitian forms on $\mathbf{C}^{4}$ defined by these matrices are equivalent, and we will write them as quadratic forms in the form

$$
\begin{aligned}
& \Phi_{0}(Z)=\left|Z^{0}\right|^{2}+\left|Z^{1}\right|^{2}-\left|Z^{2}\right|^{2}-\left|Z^{3}\right|^{2}, \\
& \Phi_{1}(Z)=-i Z^{0} \bar{Z}^{2}-i Z^{1} \bar{Z}^{3}+i Z^{2} \bar{Z}^{0}+i Z^{3} \bar{Z}^{1}, \\
& \Phi_{2}(Z)=Z^{0} \bar{Z}^{2}+Z^{1} \bar{Z}^{3}+Z^{2} \bar{Z}^{0}+Z^{3} \bar{Z}^{1} .
\end{aligned}
$$

Here $\Phi_{0}$ is the standard form, $\Phi_{1}$ is convenient for certain calculations, and $\Phi_{2}$ is the form which comes in from the spinor interpretation of certain physical quantities. We will use different forms at different times, but consider them as $\mathbf{C}^{4}$ with a particular choice of coordinates which gives the above matrices as a representation for the abstract form $\Phi$ on $C^{4}$ (or any fixed 4-complex-dimensional vector space).

Let us denote the pair $\left(C^{4}, \Phi\right)$ by $T$, the space of twistors. Let

$$
\begin{aligned}
\mathbf{T}^{+} & =\left\{Z \in \mathbf{C}^{4}: \Phi(Z)>0\right\}, \\
\mathbf{T}^{0} & =\left\{Z \in \mathbf{C}^{4}: \Phi(Z)=0\right\}, \\
\mathbf{T}^{1} & =\left\{Z \in \mathbf{C}^{4}: \Phi(Z)<0\right\} .
\end{aligned}
$$

We will call these positive, null, and negative twistors, respectively. It's clear that $\mathbf{T}=\mathbf{T}^{+} \cup \mathbf{T}^{0} \cup \mathbf{T}^{-}$, and that $\mathbf{T}^{0}$ is a real 7-dimensional cone in $\mathbf{C}^{4}$.

We now define corresponding subsets of our three basic flag manifolds $F_{12}$, $F_{1}$, and $F_{2}$. We say that $\Phi$ (subspace) $>0$, if and only if $\Phi$ (nonzero vectors in the subspace) $>0$, and similarly, $\Phi$ (subspace) $=0$ if and only if $\Phi$ (vectors in the subspace) $=0$. We want to introduce different notation for the different flag manifolds. Let

$$
\begin{aligned}
\mathbf{P}_{3} & =F_{1} \quad\left(=\mathbf{P}_{3}(\mathbf{C})\right), \\
M_{\mathbf{C}} & =F_{2} \quad\left(=G_{2,4}(\mathbf{C})\right), \\
F & =F_{12},
\end{aligned}
$$


then our basic diagram (4.1) becomes

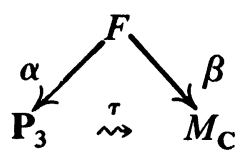

Here $\mathbf{P}_{3}$ is our basic complex manifold where complex-analytic objects will be studied, $\boldsymbol{M}_{\mathbf{C}}$ will turn out to be a complexification of compactified Minkowski space $M$, and $F$ is the auxiliary flag manifold which defines the correspondence $\tau$ between $\mathbf{P}_{\mathbf{3}}$ and $\boldsymbol{M}_{\mathbf{C}}$.

We now let

$$
\begin{aligned}
\mathbf{P}_{3}^{+} & :=\left\{L_{1} \in \mathbf{P}_{3}: \Phi\left(L_{1}\right)>0\right\}, \\
N & :=\mathbf{P}_{3}^{0}:=\left\{L_{1} \in \mathbf{P}_{3}: \Phi\left(L_{1}\right)=0\right\}, \\
\mathbf{P}_{3}^{-} & :=\left\{L_{1} \in \mathbf{P}_{3}: \Phi\left(L_{1}\right)<0\right\},
\end{aligned}
$$

Similarly, set

$$
\begin{aligned}
M_{\mathbf{C}}^{+} & :=\left\{L_{2} \in M_{\mathbf{C}}: \Phi\left(L_{2}\right)>0\right\}, \\
M & :=M_{\mathbf{C}}^{0}:=\left\{L_{2} \in M_{\mathbf{C}}: \Phi\left(L_{2}\right)=0\right\}, \\
M_{\mathbf{C}}^{-} & :=\left\{L_{2} \in M_{\mathbf{C}}: \Phi\left(L_{2}\right)<0\right\}
\end{aligned}
$$

and

$$
\begin{aligned}
F^{+} & =\left\{\left(L_{1}, L_{2}\right) \in F: \Phi\left(L_{2}\right)>0\right\}, \\
F^{0} & =\left\{\left(L_{1}, L_{2}\right) \in F: \Phi\left(L_{2}\right)=0\right\}, \\
F^{-} & =\left\{\left(L_{1}, L_{2}\right) \in F: \Phi\left(L_{2}\right)<0\right\} .
\end{aligned}
$$

Here we have $N=$ projective null twistors, and $M$ is the null 4-dimensional subspace of $\mathbf{C}^{4}$, which, as we shall see, will turn out to be compactified Minkowski space.

We have induced correspondences

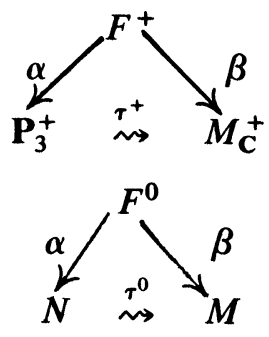

(and we omit, by symmetry, any further discussion of $F^{-}$, etc., since it will be the same as the discussion of $F^{+}$, etc.).

We want to describe the geometric nature of each of the spaces above; and then we will look at the induced correspondences. We will summarize the basic geometric properties of the above spaces in the following proposition.

4.6 Proposition. (1) $\mathbf{P}_{3}^{+}$contains a 4-complex dimensional family of projective complex lines parametrized by $M_{\mathbf{C}}^{+}$. 
(2) $M_{\mathbf{C}}^{+}$is biholomorphically equivalent to the (bounded symmetric) domain of $2 \times 2$ complex matrices whose Hermitian imaginary part is positive definite.

(3) Let $M_{0}$ be the Hermitian $2 \times 2$ matrices, then $M_{0}$ is a boundary component of $M_{\mathbf{C}}^{+}$, and is an open dense subset of $M$.

(4) $M$ is a compact 4-dimensional real-analytic submanifold of $M_{\mathbf{C}}$ which is diffeomorphic to $S^{1} \times S^{3}$.

(5) $N$ is a compact 5-dimensional real-analytic hypersurface in $\mathbf{P}_{\mathbf{3}}$ diffeomorphic to $S^{2} \times S^{3}$.

(6) $F^{+}$is biholomorphic to $\mathbf{P}_{1} \times M_{\mathbf{C}}^{+}$.

This will not be proved in detail in this paper, but we will discuss the ideas of the proof briefly. (1) is clear from the correspondence $\tau$. (4) and (5) follow most simply from the homogeneous space representation of $M_{\mathrm{C}}^{+}, M$ and $N$ in $\S 5$. We will give an elementary representation of $M_{\mathbf{C}}^{+}$in terms of matrices from which (2), (3) and (6) will be simple consequences. Namely, let $\Phi$ be represented by

$$
\Phi_{1}=\left[\begin{array}{cc}
0 & i I_{2} \\
-i I_{2} & 0
\end{array}\right],
$$

then consider the mapping

$$
z=\left(z^{i j}\right)=\left[\begin{array}{ll}
z^{11} & z^{12} \\
z^{21} & z^{22}
\end{array}\right] \stackrel{A}{\rightarrow}\left\{\text { subspace spanned by }\left[\begin{array}{c}
z \\
I_{2}
\end{array}\right]\right\} .
$$

Then $A: \mathbf{C}^{4} \rightarrow M_{\mathbf{C}}$ is an affine coordinate system on an open dense subset of $M_{\mathrm{C}}$. We have chosen $\Phi_{1}$ so that $M_{\mathrm{C}}^{+}$is contained entirely in $M_{\mathrm{C}}^{A}=A\left(\mathrm{C}^{4}\right) \subset$ $M_{\mathbf{C}}$, the affine part of $M_{\mathbf{C}}$. Now, letting $\gg$ denote positive definite,

$$
\begin{aligned}
\Phi_{1}(A(z)) & >0 \Leftrightarrow\left[z^{*}, I_{2}\right]\left[\begin{array}{cc}
0 & +i I_{2} \\
-i I_{2} & 0
\end{array}\right]\left[\begin{array}{c}
z \\
I_{2}
\end{array}\right] \gg 0, \\
& \Leftrightarrow-i\left(z-z^{*}\right) \gg 0 \\
& \Leftrightarrow \operatorname{Im} z \gg 0, \quad \text { where } \operatorname{Im} z=\left(z-z^{*}\right) / 2 i,
\end{aligned}
$$

and it follows that $M_{\mathbf{C}}^{+} \cong\{2 \times 2$ matrices $z$ with $\operatorname{Im} z \gg 0\}$. The Hermitian $2 \times 2$ matrices, $H(2)$, can be identified with the set of $2 \times 2$ matrices satisfying $\operatorname{Im} z=0$, a boundary component of $M_{\mathrm{C}}^{+}$. We denote $M_{0}=$ $A(H(2))$ under the affine coordinate mapping. Then clearly $M_{0} \subset M$ since $\Phi(A(H(2))) \equiv 0$, by the same computation as above. It follows easily that $M_{\mathrm{C}}^{A} \cap M=M_{0}$ is open and dense in $M$.

We now look at the correspondences $\tau^{+}$and $\tau^{0}$ in (4.4) and (4.5).

4.8 Proposition. (1) $\tau^{+}(p)$ is the intersection of an affine complex 2-plane with $M_{\mathrm{C}}^{+}$.

(2) $\tau^{0}(p)$ is a circle $S^{1}$ embedded in $M$.

(3) $\left(\tau^{+}\right)^{-1}(p)$ and $\left(\tau^{0}\right)^{-1}(p)$ are complex projective lines embedded in $\mathbf{P}_{3}^{+}$and $N$ respectively. 
Proof. (1) is just the restriction of $\tau(p)$ to $M_{\mathrm{C}}^{+}$which is easily seen to be the intersection of an affine 2-plane with $M_{\mathbf{C}}^{+}$by using the affine coordinates representation $\mathbf{C}^{4} \stackrel{A}{\rightarrow} M_{\mathbf{C}}$ introduced earlier. Let's look at part (2), and let's use the coordinate mapping (4.7) above. But we will use

$$
\Phi_{0}=\left[\begin{array}{cc}
I_{2} & 0 \\
0 & -I_{2}
\end{array}\right]
$$

to represent the Hermitian form $\Phi$ on $\mathbf{C}^{4}$. Let

$$
v=\left[\begin{array}{l}
1 \\
0 \\
1 \\
0
\end{array}\right] \operatorname{span} L_{1} \subset \mathbf{C}^{4},
$$

and we see that $\Phi_{0}(v)=v^{*} \Phi_{0} v=0$, so $\Phi\left(L_{1}\right)=0$. Then $\left\{L_{2}: L_{1} \subset L_{2}\right\}$ is parametrized by

$$
\left[\begin{array}{ll}
1 & z^{12} \\
0 & z^{22}
\end{array}\right] \stackrel{A}{\rightarrow} \operatorname{span}\left[\begin{array}{ll}
1 & 0 \\
0 & 1 \\
1 & z^{12} \\
0 & z^{22}
\end{array}\right] .
$$

Now we require that $\Phi\left(L_{2}\right)=0$ also. This implies that if

$$
v_{2}=\left[\begin{array}{c}
0 \\
1 \\
z^{12} \\
z^{22}
\end{array}\right],
$$

then

$$
\Phi_{0}\left(v_{2}\right)=v_{2}^{*} \Phi_{0} v_{2}=0
$$

i.e. that $\left|z^{12}\right|^{2}+\left|z^{22}\right|^{2}=1$. But we also have

$$
\Phi_{0}\left(\alpha v_{1}+\beta v_{2}\right)=0, \text { for all } \alpha, \beta \in \mathbf{C},
$$

so this implies easily that $z^{12}=0$, and we have

$$
\left[\begin{array}{cc}
1 & 0 \\
0 & 1 \\
1 & 0 \\
0 & e^{i \theta}
\end{array}\right]
$$

parametrizes all of the 2-planes $L_{2} \supset L_{1}$ with $\Phi\left(L_{2}\right)=0$. This is a circle, completely contained in this particular affine coordinate system. The choice of the initial vector $v_{1}$ is immaterial, by the homogeneity of the null twistors with respect to $U(2,2)$ (cf. §5). Q.E.D.

We recall the basic diagram (4.3)

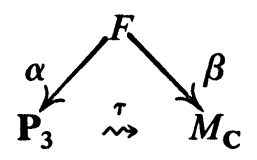


with the correspondence $\tau$, which we will call the Penrose correspondence, since almost all of the properties in this paper dealing with this correspondence are due to Penrose, and were developed in a series of papers, originating in [17]. We have modified Penrose's presentation by introducing the auxiliary flag manifold $F$ to facilitate the discussion of the correspondence in Penrose's approach to these problems. It will play a more important role later when we discuss the cohomology representation of physical fields.

We state now our final geometric proposition of this section. Let $Z$ and $W$ be twistors in $T$, and so $[Z]$ and $[W]$ are well defined points in $\mathbf{P}_{3}$. Then define

$$
\tau(Z)=\tau([Z])
$$

the corresponding 2-plane in $M_{\mathbf{C}}$.

4.9 Proposition. (1) $\tau(Z) \cap \tau(W)=\{$ point $\} \in M_{\mathbf{C}}$ unless $[Z]=[W]$;

(2) $\tau(Z) \cap \tau(W) \in M$ if and only if $\Phi(Z)=\Phi(W)=0$ and $\Phi(Z, W)=0$.

The proof is not difficult, and is omitted (cf. Penrose [17]).

5. Homogeneous spaces and group actions. In this section we want to discuss the homogeneous space structure of the flag manifolds studied in the previous section. The complex Lie group $G_{\mathbf{C}}=S L(4, \mathrm{C})$ acts transitively on $\mathrm{C}^{4}$, and induces a transitive action on $F, P_{3}$ and $M_{\mathbf{C}}$. Reverting to our notation at the beginning of $\S 4$ we have $G_{\mathrm{C}}$ acting on $F_{12}, F_{1}$, and $F_{2}$. Let $P_{12}, P_{1}$ and $P_{2}$ be the subgroup of $G_{C}$ which leaves a point of $F_{12}, F_{1}$ and $F_{2}$ fixed. These subgroups are isomorphic to subgroups of the form

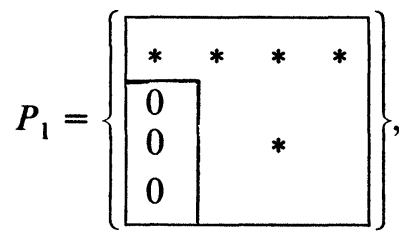

$$
\begin{aligned}
& P_{12}=\left\{\left[\begin{array}{c|ccc}
* & * & * & * \\
\hdashline 0 & * & * & * \\
0 & 0 & * & * \\
0 & 0 & * & *
\end{array}\right]\right\} \text {, } \\
& P_{2}=\left\{\left[\begin{array}{c|ccc}
* & * & * & * \\
\hdashline * & * & * & * \\
\cline { 1 - 2 } & 0 & * & * \\
0 & 0 & * & *
\end{array}\right]\right\},
\end{aligned}
$$

So $F_{12}=G_{\mathrm{C}} / P_{12}, F_{1}=G_{\mathrm{C}} / P_{1}, F_{2}=G_{\mathrm{C}} / P_{2}$.

Now $F_{12}^{+}, F_{1}^{+}$, and $F_{2}^{+}$are all three open orbits in the above compact complex homogeneous manifolds under the action of the real form $S U(2,2)$ of $S L(4, \mathrm{C})$. Namely, any 2-dimensional subspace $L_{2} \subset \mathrm{C}^{4}$ which is positive 
(i.e. $\Phi\left(L_{2}\right)>0$ ), can be mapped to a given 2-dimensional positive subspace by an element of $S U(2,2)$. This can be seen by taking a basis for $L_{2}$ orthonormal with respect to $\Phi$, and extending it to a $\Phi$-orthonormal basis for $\mathbf{C}^{4}$, doing the same for the second subspace, and the mapping sending one basis to another will necessarily be in $U(2,2)$. Dividing by the determinant of this matrix gives the desired mapping.

The open orbits are special cases of open homogeneous complex manifolds which have been objects of considerable study in the past decades (cf. [33], where there is a detailed discussion of such "flag domains," with additional references). We see immediately if we let $G=S U(2,2)$, then

$$
F_{12}^{+}=G / P_{12} \cap G, \quad F_{1}^{+}=G / P_{1} \cap G, \quad F_{2}^{+}=G / P_{2} \cap G,
$$

which turn out to be

$$
\begin{aligned}
\mathbf{P}_{3}^{+} & =S U(2,2) / S(U(1) \times U(1,2))=U(2,2) / U(1) \times U(1,2), \\
F^{+} & =S U(2,2) / S(U(1) \times U(1) \times U(2))=U(2,2) / U(1) \times U(1) \times U(2), \\
M_{\mathbf{C}}^{+} & =S U(2,2) / S(U(2) \times U(2))=U(2,2) / U(2) \times U(2),
\end{aligned}
$$

where $S()$ means determinant $=1$, and we have written down a second representation on the right hand side. It's not clear which group-theoretic representation is most useful at this stage of development. Of interest is that the isotropy subgroup for $\mathbf{P}_{3}^{+}$is not compact, whereas much of the machinery of group representation, and homogeneous manifold theory depend on having compact isotropy subgroups (cf. Wells-Wolf [33], and the references cited there). We note that $M_{\mathbf{C}}^{+}$above is of the form (semisimple group)/ (maximal compact), which means it is an Hermitian symmetric space, and hence a bounded symmetric domain, namely the tube domain we saw in $\$ 4$.

The main vector bundles of interest on these homogeneous spaces will be the tangent bundle and the powers of the hyperplane section bundle of $\mathbf{P}_{3}$ (cf. $\S 8$ ). These are all representable as homogeneous vector bundles on $\mathbf{P}_{3}^{+}$ induced by representations of the isotropy subgroup, which we won't dwell on here, but which is important in certain contexts.

Also, we have a homogeneous representation for the compact manifolds which are also orbits of $G=S U(2,2)$, but closed orbits, and hence minimal dimensional (cf. Wolf [34]). Namely,

$$
N=S U(2,2) / P_{N},
$$

where

$$
P_{N} \cong\left[\begin{array}{ccc}
a & b & c \\
0 & U(1,1) & d \\
0 & 0 & e
\end{array}\right] \subset S U(2,2)
$$

where $a, b, c, d, e$ are appropriate complex vectors and numbers satisfying the orthogonality relation of $S U(2,2) . P_{N}$ contains two subgroups, the conformal- $U(1,1)$ group

$$
\left[\begin{array}{ccc}
a & 0 & 0 \\
0 & U(1,1) & 0 \\
0 & 0 & b
\end{array}\right], \quad \operatorname{det}[]=1,
$$


as well as the Heisenberg group

$$
\left[\begin{array}{llll}
1 & b_{1} & b_{2} & c \\
0 & 1 & 0 & \overline{b_{1}} \\
0 & 0 & 1 & \overline{b_{2}} \\
0 & 0 & 0 & 1
\end{array}\right], \quad c \text { real. }
$$

See Chern and Moser [5] or Tanaka [27] for a good description of the geometry of such hyperquadrics defined by Hermitian quadratic forms of mixed signature. The surface $M$ is a similar hyperquadric in $M_{\mathbf{C}}$, and has the form

$$
M=S U(2,2) / P_{M},
$$

where $P_{M}$ is a different real parabolic subgroup, namely $\boldsymbol{P}_{\boldsymbol{M}}$ has the form:

$$
P_{M} \cong\left[\begin{array}{ccc}
A & B \\
0 & 0 & \\
0 & 0 & C
\end{array}\right] \subset S U(2,2) .
$$

We will not be making use of the specific nature of the groups which enter here later in this paper, but we recall that the topological type of $N$ and $M$ are determined by this homogeneous structure (cf. Wolf [34]). One can also see the topological nature of $N$ and $M$ by using more elementary methods (cf. Penrose [17]), but we leave this for the reader to sort out.

We now want to look at the action on $M_{\mathbf{C}}^{+}$and $M_{0}$ induced by $S U(2,2)$. Suppose that we represent $\Phi$ by

$$
\Phi_{1}=\left[\begin{array}{cc}
0 & i I_{2} \\
-i I_{2} & 0
\end{array}\right]
$$

as before. Then any $g \in S U(2,2)$ can be expressed as

$$
g=\left[\begin{array}{ll}
A & B \\
C & D
\end{array}\right], \quad A, B, C, D \text { each } 2 \times 2 \text { complex matrices, }
$$

and the coefficients of $g$ must satisfy the orthogonality relation

$$
g^{*} \Phi_{1} g=\Phi_{1}
$$

which becomes

$$
A B^{*}=B A^{*}, A D^{*}-B C^{*}=I_{2}, C D^{*}=D C^{*},
$$

and $\operatorname{det} g=1$.

Now choose affine coordinates $\left[{ }_{I}^{z}\right]$, for $M_{\mathrm{C}}$ as before, where $z=\left(z^{i j}\right)$ is a $2 \times 2$ matrix. Then the action on the "homogeneous coordinates" for $M_{\mathbf{C}}$

$$
\left[\begin{array}{l}
z_{1} \\
z_{2}
\end{array}\right], \quad z_{1}, z_{2} \quad 2 \times 2 \text { matrices, }
$$

is given by

$$
g\left[\begin{array}{l}
z_{1} \\
z_{2}
\end{array}\right]=\left[\begin{array}{ll}
A & B \\
C & D
\end{array}\right]\left[\begin{array}{l}
z_{1} \\
z_{2}
\end{array}\right]=\left[\begin{array}{l}
A z_{1}+B z_{2} \\
C z_{1}+D z_{2}
\end{array}\right]
$$


This induces on the affine coordinates the mapping

$$
z \rightarrow(A z+B)(C z+D)^{-1},
$$

which is only well defined where $(C z+D)$ is invertible (an open dense set of $\left.M_{\mathrm{C}}^{A}\right)$.

Let $d s^{2}:=\operatorname{det}(d z)$ be a holomorphic metric form on $M_{\mathrm{C}}^{A}$. This corresponds to the Minkowski metric in $M_{0}$ (cf. §2) extended to the complexification $M_{\mathrm{C}}^{A}$ of $M_{0}$ in a C-linear manner. Now look at the subgroup of $G=S U(2,2)$ given by matrices of the form

$$
\tilde{P}=\left\{\left[\begin{array}{cc}
A & B \\
0 & D
\end{array}\right]\right\}
$$

Then necessarily

$$
D=\left(A^{*}\right)^{-1}, A B^{*}=B A^{*} \text { and } \operatorname{det}\left(A\left(A^{*}\right)^{-1}\right)=1 .
$$

The action of $\tilde{P}$ on $M_{\mathrm{C}}^{A}$ is given by

$$
z \rightarrow(A z+B)\left(\left(A^{*}\right)^{-1}\right)^{-1}=A z A^{*}+B A^{*} .
$$

If we let $P \subset \tilde{P}$ be defined by

$$
P=\left\{\left[\begin{array}{cc}
A & B \\
0 & D
\end{array}\right], A \in S L(2, \mathbf{C})\right\},
$$

then we see that

$$
z \rightarrow A z A^{*}+B A^{*}
$$

consists of the action of $S L(2, \mathrm{C})$ on $M_{0}$ given in $\$ 3$, plus the translations by the Hermitian matrix $B A^{*}$, which is precisely the Poincare group acting on $M_{0}$, extended in a $\mathbf{C}$-linear manner to $M_{\mathrm{C}}^{A}$. In particular, if $z=z^{*}$, i.e. is Hermitian, then $P$ does act as the Poincaré group on $M_{0}$. Moreover these mappings preserve $d s^{2}$.

We see that the dilations correspond to mappings of the form

$$
g_{\rho}=\left[\begin{array}{ll}
\rho & 0 \\
0 & \rho^{-1}
\end{array}\right] \subset \tilde{P}, \quad \rho \in \mathbf{R}
$$

then

$$
g_{\rho}(z)=\rho^{2} z,
$$

and $\tilde{P} / P$ is precisely the dilations. The inversions are given by the mappings in $S U(2,2)$ of the form

$$
\left\{\left[\begin{array}{ll}
0 & B \\
C & 0
\end{array}\right], B C^{*}=-I\right\} \text { or }\left\{\left[\begin{array}{cc}
0 & B \\
\left(-B^{*}\right)^{-1} & 0
\end{array}\right]\right\} .
$$


i.e.

$$
z \rightarrow B\left[\left(-B^{*}\right)^{-1} z\right]^{-1}=-B z^{-1} B^{*} .
$$

If we let $g(z)=-B z^{-1} B^{*}$, then

$$
\begin{aligned}
g^{*}\left(d s^{2}\right) & =\operatorname{det}(d g(z)) \\
& =\operatorname{det}\left(-B d\left(z^{-1}\right) B^{*}\right) \\
& =\operatorname{det}\left(-B\left[-z^{-1} d z \cdot z^{-1}\right] B^{*}\right) \\
& =\operatorname{det}\left(P B^{*}\right)(\operatorname{det} z)^{-2} \operatorname{det}(d z) \\
& =\operatorname{det}\left(B^{*}\right)(\operatorname{det} z)^{-2} d s^{2},
\end{aligned}
$$

which is a multiple of the original metric form $d s^{2}$, so the action by $g$ is a conformal mapping of the Minkowski space. We remark that the conformal factor in $M_{0}$ is a positive scalar function and in $M_{\mathrm{C}}^{A}$ it is the square of a holomorphic function of $z$ which restricts to a positive conformal factor on $M_{0}$.

Thus we have a conformal metric (metric defined up to conformality) at each point of $M$, which agrees with the usual Minkowski metric (up to conformality) at each point of $M_{0}$, since $M_{\mathrm{C}}^{A}$ is a typical model of an affine coordinate system on $\boldsymbol{M}_{\mathbf{C}}$.

We recall now that if $Z \neq 0$ in $T$, then

$$
\tau(Z) \cong \mathbf{P}_{2} \subset M_{\mathrm{C}}, \quad \tau^{0}(Z) \cong S^{1} \subset M .
$$

5.1 Proposition. (1) The plane $\tau(Z)$ is null with respect to the conformal Minkowski metric $d s^{2}$ on $M_{\mathrm{C}}$, i.e., each tangent vector to $\tau(Z)$ is a null vector.

(2) The circle $\tau^{0}(Z)$ is null with respect to the conformal Minkowski metric on M.

Proof. Let $Z \neq 0$ be given, and suppose that $Z=(1,0, \alpha, \beta)$, which is no loss of generality, and then $\tau(Z)$ is parametrized by

$$
\left\{L_{2} \supset L_{1}=[Z]\right\} \sim \operatorname{span}\left[\begin{array}{ll}
1 & 0 \\
0 & 1 \\
\alpha & z^{12} \\
\beta & z^{22}
\end{array}\right] \sim\left[\begin{array}{ll}
\alpha & z^{12} \\
\beta & z^{22}
\end{array}\right]
$$

Thus we have a mapping

$$
\begin{aligned}
& \mathbf{C}^{2} \stackrel{T}{\rightarrow} \mathbf{C}^{4} \cong M_{\mathrm{C}}^{A}, \\
&\left(z^{12}, z^{22}\right) \stackrel{T}{\mapsto}\left[\begin{array}{ll}
\alpha & z^{12} \\
\beta & z^{22}
\end{array}\right] .
\end{aligned}
$$

We see that

$$
T^{*}\left(d s^{2}\right)=\operatorname{det}\left[\begin{array}{ll}
0 & d z^{12} \\
0 & d z^{22}
\end{array}\right]=0
$$


and the image of $T$ is clearly a null 2-plane. Case (2) is proved in exactly the same manner. Q.E.D.

The following proposition is a basic property of the Penrose correspondence.

5.2 Proposition. Let $q_{1}$ and $q_{2}$ be two points in $M$, then $q_{1}$ and $q_{2}$ can be joined by a null curve (are null-separated) if and only if $\tau_{0}^{-1}\left(q_{1}\right) \cap \tau_{0}^{-1}\left(q_{2}\right) \neq \varnothing$ in $N$.

Proof. Let $p=\tau_{0}^{-1}\left(q_{1}\right) \cap \tau_{0}^{-1}\left(q_{2}\right)$, then $\tau(p)$ is a null curve in $M$ which meets $q_{1}$ and $q_{2}$. What remains to be shown is that any null curve in $M$ corresponds to a point in $N$. By restricting attention to $M_{0}$, we can consider null straight lines (light rays), and a given light ray will determine by simple linear algebra a nonzero null twistor in $T$ which determines a specific point in $N$. It's easiest to express in terms of spinors, which are developed in the next section, and we refer the reader to Penrose [17] for a complete proof of this proposition. Q.E.D.

6. Tensors and spinors. If $M$ is a Riemannian or pseudo-Riemannian manifold with a metric $g$ given locally by $d s^{2}=g_{a b} d x^{a} d x^{b}$, then the metric gives an isomorphism

$$
T(M) \cong_{g} T^{*}(M),
$$

and if $v=v^{a} \partial / \partial x^{a}, w=w_{b} d x^{b}$, then the isomorphism is explicitly given by identifying $v$ with coefficients $\left\{v^{a}\right\}$ with $w$ with coefficients $v_{b}=v^{a} g_{a b}$. We have used the summation convention throughout, and this process is known as "lowering and raising indices," and is a means of shifting from tangent vectors to cotangent vectors by means of the metric $g$.

In general a tensor

$$
T=T_{\text {def } \cdots}^{a b c \cdots} \frac{\partial}{\partial x^{a}} \otimes \frac{\partial}{\partial x^{b}} \otimes \frac{\partial}{\partial x^{c}} \otimes \cdots \otimes d x^{d} \otimes d x^{e} \otimes d e^{f} \ldots
$$

is a section of

$$
T(M) \otimes T(M) \otimes \cdots \otimes T^{*}(M) \otimes \cdots \otimes T^{*}(M),
$$

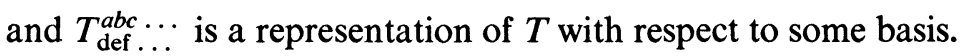

The spinor calculus is a generalization of the tensor calculus, with the addition of a certain amount of complex structure. We will give a simple version of it adequate for our purposes (for more details, see [28]). Consider

$$
\mathbf{S}=\left(\mathbf{C}^{2}, \varepsilon\right)
$$

where $\varepsilon$ is a skew-symmetric nondegenerate complex bilinear form. $\mathbf{S}$ is the basic space of spinors, from which higher order spinors are derived (just as the tangent space equipped with an inner product induces all higher order Riemannian tensors). Since $\varepsilon$ is nondegenerate, we can use $\varepsilon$ to identify $\mathbf{S}$ with $\mathbf{S}^{*}$, the complex-linear dual of $\mathbf{S}$. We have

$$
\begin{aligned}
\mathbf{S} \otimes_{\mathbf{R}} \mathbf{C} & =\mathbf{S}^{1,0} \oplus \mathbf{S}^{0,1}, \\
\mathbf{S}^{*} \otimes_{\mathbf{R}} \mathbf{C} & =\mathbf{S}_{1,0}^{*} \oplus \mathbf{S}_{0,1}^{*},
\end{aligned}
$$

where $\mathbf{S}^{1,0}$, and $\mathbf{S}^{0,1}$ are the $+i$ and $-i$ eigenspaces of the almost-complex 
structure $\boldsymbol{J}$ of $\mathbf{S}$ (multiplication by $\boldsymbol{i}$ considered as an $\mathbf{R}$-linear mapping in $\mathbf{C}$ ), extended in a $\mathbf{C}$-linear fashion to $\mathbf{S} \otimes_{\mathbf{R}} \mathbf{C}$. We treat $\mathbf{S}^{*} \otimes \mathbf{C}$ in the same fashion. Now extend $\varepsilon$ to $\mathbf{S} \otimes \mathbf{C}$ by $\mathbf{C}$-linearity, and we can identify $\mathbf{S}^{1,0}$ with $\mathbf{S}_{1,0}^{*}$ by means of $\varepsilon$ also. Note that $S$ is complex-linearly equivalent to $\mathbf{S}^{1,0}$ and that $\mathbf{S}$ is conjugate-complex-linear equivalent to $\mathbf{S}^{0,1}$.

A spinor is an element of the tensor product of some combination of the above four spaces, e.g.

$$
\mathbf{S}^{1,0} \otimes \mathbf{S}^{0,1} \otimes \mathbf{S}_{1,0}^{*} \otimes \mathbf{S}_{0,1}^{*}
$$

We can choose a basis

$$
\left\{e_{A}, A=0,1\right\} \text { for } \mathbf{C}^{2}
$$

so that

$$
\varepsilon=\left[\varepsilon_{A B}\right]=\left[\begin{array}{cc}
0 & 1 \\
-1 & 0
\end{array}\right]
$$

in those coordinates. Then a spinor $\xi \in \mathbf{S}^{1,0}$ can be represented as

$$
\xi=\xi^{A} e_{A}, \quad \xi^{A} \in \mathbf{C}, A=0,1,
$$

or $\xi \in \mathbf{S}^{1,0} \otimes \mathbf{S}^{0,1}$ can be represented as

$$
\xi=\xi^{A \bar{B}} e_{A} \otimes \overline{e_{B}},
$$

where $\left\{\overline{e_{B}}=e_{\bar{B}}\right\}$ is a basis for $\mathbf{S}^{0,1}$. For instance in the usual complex analyst's notation for $\mathbf{C}^{2}$, we would have

$$
\left\{e_{A}\right\}=\left\{\frac{\partial}{\partial z^{0}}, \frac{\partial}{\partial z^{1}}\right\}, \quad\left\{\overline{e_{A}}\right\}=\left\{\frac{\partial}{\partial \bar{z}^{0}}, \frac{\partial}{\partial \bar{z}^{1}}\right\},
$$

with the dual basis

$$
\left\{e^{A}\right\}=\left\{d z^{0}, d z^{1}\right\}, \quad\left\{\overline{e^{A}}\right\}=\left\{\overline{d z^{0}}, \overline{d z^{1}}\right\} .
$$

A more general spinor might be

$$
\xi=\xi_{B}^{A} \bar{C}_{\bar{D}} e_{A} \otimes e^{B} \otimes \overline{e_{C}} \otimes \overline{e^{D}} .
$$

These spinors were originally developed by van der Waerden, and the majority of the physics literature uses $\zeta^{\bar{A}}=\zeta^{A}$, whereas Penrose and his school uses $\xi^{\bar{A}}=\xi^{A^{\prime}}$, since this is easier on the typesetter. We will adopt Penrose's index notation (which can be considered as coefficients as we've done here, or as abstract spinors, sections of $\mathbf{S}^{1,0} \otimes \mathbf{S}_{0,1}^{*} \ldots$, where the indices label which tensor products are involved, cf. [18]). To raise or lower indices, we use the formulas

$$
\xi_{B}=\xi^{A} \varepsilon_{A B}, \quad \xi^{A}=\varepsilon^{A B} \xi_{B},
$$

noting that $\varepsilon_{A B}$ is skew symmetric, and that the order is important. For instance if

$$
\xi^{A}=\left[\begin{array}{l}
\xi^{0} \\
\xi_{1}
\end{array}\right]
$$


then $\xi^{0}=\xi_{1}, \xi^{1}=\xi_{0}$, i.e.

$$
\begin{aligned}
{\left[\begin{array}{r}
\xi_{1} \\
-\xi_{0}
\end{array}\right] } & =\left[\begin{array}{rr}
0 & 1 \\
-1 & 0
\end{array}\right]\left[\begin{array}{l}
\xi_{0} \\
\xi_{1}
\end{array}\right], \\
\xi^{A} & =\varepsilon^{A B} \cdot \xi_{B} .
\end{aligned}
$$

We can consider $M_{0} \times \mathscr{T}(\mathbf{S})$, where $\mathscr{T}(\mathbf{S})$ is the tensor algebra of spinors, and have spinors at points of Minkowski space. Moreover, there is a mapping

$$
T\left(M_{\mathrm{C}}^{A}\right)_{0} \stackrel{\rightrightarrows}{\cong} \mathrm{S}^{1,0} \otimes \mathrm{S}^{0,1}
$$

where $M_{\mathrm{C}}^{A}$ is affine complexified Minkowski space (= $2 \times 2$ complex matrices), given by sending

$$
x^{a} \rightarrow\left[\begin{array}{ll}
u & \zeta \\
\bar{\zeta} & v
\end{array}\right]=\left[\begin{array}{ll}
p^{00^{\prime}} & p^{01^{\prime}} \\
p^{10^{\prime}} & p^{11^{\prime}}
\end{array}\right]=x^{A A^{\prime}},
$$

Here $x^{a}$ is a coordinate representation of a holomorphic tangent vector at 0 in $M_{\mathbf{C}}^{A}$, with respect to a frame for which the metric $g$ on Minkowski space $M_{0}$ is of the form $d s^{2}=\left(d x^{0}\right)^{2}-\left(d x^{1}\right)^{2}-\left(d x^{2}\right)^{2}-\left(d x^{3}\right)^{2}$. We recall that

$$
\begin{array}{ll}
u=\frac{1}{\sqrt{2}}\left(x^{0}+x^{1}\right), & \zeta=\frac{1}{\sqrt{2}}\left(x^{2}+i x^{3}\right), \\
\bar{\zeta}=\frac{1}{\sqrt{2}}\left(x^{2}-i x^{3}\right), & v=\frac{1}{\sqrt{2}}\left(x^{0}-x^{1}\right) .
\end{array}
$$

Now Lorentz transformations on $x^{a}$ induce a mapping by $S L(2, \mathrm{C})$ acting on the $2 \times 2$ matrix $x^{A A^{\prime}}$ given by

$$
\left[x^{A A^{\prime}}\right] \rightarrow S\left[x^{A A^{\prime}}\right] S^{*}, \quad S \in S L(2, \mathrm{C}),
$$

as in $\S 3$. The action of $S L(2, \mathrm{C})$ on the matrix $x^{A A^{\prime}}$ is just the action of $S L(2, \mathbf{C})$ on $\mathbf{S}^{1,0} \otimes \mathbf{S}^{0,1}$, induced from the action of $S L(2, \mathbf{C})$ on $\mathbf{C}^{2}$. Moreover this action preserves the skew-symmetric form $\varepsilon$, and hence the full spinor algebra. Thus we have an embedding of tensor algebras (letting $\mathscr{T}\left(M_{0}\right)=$ the full tensor algebra of Minkowski space)

$$
\mathscr{T}\left(M_{\mathbf{C}}^{A}\right)_{0} \rightarrow M_{\mathbf{C}}^{A} \times \mathcal{T}(\mathbf{S})
$$

which is $\operatorname{SL}(2, \mathbf{C})$ equivariant, and is induced by the mapping

$$
x^{a} \rightarrow x^{A A^{\prime}}
$$

taking $T\left(M_{\mathbf{C}}^{A}\right) \rightarrow \mathbf{S}^{1,0} \otimes \mathbf{S}^{0,1}$. We have the diagram

$$
\begin{aligned}
& \begin{array}{ccc}
\mathscr{T}\left(M_{\mathbf{C}}^{A}\right)_{0} & \rightarrow & \mathbf{S}^{1,0} \otimes \mathbf{S}^{0,1} \\
\uparrow & \uparrow
\end{array} \\
& T\left(M_{0}\right)_{0} \rightarrow\left\{x^{A A^{\prime}}=\overline{x^{A^{\prime} A}}\right\} \quad \text { (Hermitian), }
\end{aligned}
$$

where we use the convention that $\overline{\xi^{A}}=\bar{\xi}^{\mathrm{A}^{\prime}}$, just as in the calculus of differential forms one writes $w=f(z) d z$, and $\bar{w}=\overline{f(z)} d \bar{z}$. We have a means therefore of transforming [tensor algebra objects] into [spinor algebra objects]. The spinor algebra is richer, and has more structure, and it simplifies many geometric considerations. There is an analogy to introducing the 
complex numbers as a larger and richer field than the real numbers. We will be principally interested in tensor fields, i.e. sections of the tensor bundle under consideration, all bundles being bundles over $\boldsymbol{M}_{0}$ or $\boldsymbol{M}_{\mathbf{C}}^{a}$. These bundles are trivial as global bundles, and there are corresponding nontrivial bundles for $M$ or $M_{\mathbf{C}}$, which we do not want to introduce at this time since we will not need them in this paper.

Physical quantities in (special relativity) physics are usually described in terms of tensor fields over $\boldsymbol{M}_{\mathbf{0}}$. Scalar fields are invariants, and one considers vector fields, differential forms, etc. All of these can be expressed in terms of spinors by the above embedding, e.g. a metric form $g_{a b}$ would become

$$
g_{a b} \rightarrow g_{A A^{\prime} B B^{\prime}}
$$

etc. (cf. [28]).

One reason for introducing spinors is that we can "take square roots" of null vectors. This is important if the null vector in question is of a quadratic nature, e.g. a momentum vector.

6.1 Proposition. Let $v^{a}$ be a null vector, then letting $v^{a} \rightarrow v^{A A^{\prime}}$, there exists spinors $\xi^{A}, \tau^{A^{\prime}}$ so that

$$
v^{A A^{\prime}}=\xi^{A} \tau^{A^{\prime}},
$$

and if $v^{a}$ is real, then there exists $\xi^{A}$ so that

$$
v^{A A^{\prime}}=\xi^{A} \bar{\xi}^{A^{\prime}}
$$

Proof. Set up the equation

$$
\left[\begin{array}{ll}
v^{00^{\prime}} & v^{01^{\prime}} \\
v^{10^{\prime}} & v^{11^{\prime}}
\end{array}\right]=\left[\begin{array}{ll}
\xi^{0} \tau^{0^{\prime}} & \xi^{0} \tau^{1^{\prime}} \\
\xi^{1} \tau^{0^{\prime}} & \xi^{1} \tau^{1^{\prime}}
\end{array}\right]
$$

and solve! It's clear that det $=0$ is a necessary condition to have a solution, for a given $v^{A A^{\prime}}$. It's also sufficient. Q.E.D

We can now think of $\left\{x^{a}\right\}$ coordinates in $M_{0}$ or $x^{A A^{\prime}}$ as coordinates in $M_{0}$, by the above conventions. Since these are good coordinates, we may consider $\partial / \partial x^{A A^{\prime}}$, and other combinations of derivatives as a means of expressing differential equations.

7. Maxwell's equations and the zero-rest-mass field equations. We now want to study Maxwell's equations. Classically they take the form

$$
\begin{aligned}
\frac{\partial B}{\partial t}+\operatorname{curl} E & =0, & E & =\text { electric field, } \\
\frac{\partial E}{\partial t}-\operatorname{curl} B & =j, & B & =\text { magnetic field, } \\
\operatorname{div} B & =0, & j & =\text { current } \\
\operatorname{div} E & =\sigma, & \sigma & =\text { charge density. }
\end{aligned}
$$

These equations are invariant with respect to Lorentz transformations, where we set $t=x^{0}$. We shall concern ourselves only with the homogeneous Maxwell's equations, where $j=\sigma=0$, and we will refer to the homogeneous equations simply as Maxwell's equations. We want to rewrite these equations 
in a form such that the Penrose correspondence gives us a holomorphic representation of solutions. We will do this in a sequence of steps.

First we define a Maxwell 2-tensor or 2-form, by defining the skewsymmetric matrix

$$
\left[F_{a b}\right]:=\left[\begin{array}{cccc}
0 & -E_{1} & -E_{2} & -E_{3} \\
E_{1} & 0 & B_{3} & -B_{2} \\
E_{2} & -B_{3} & 0 & B_{1} \\
E_{3} & B_{2} & -B_{1} & 0
\end{array}\right]
$$

and setting

$$
F=F_{a b} d x^{a} \wedge d x^{b} .
$$

This is a 2-form in Minkowski space with coordinates $x^{0}, x^{1}, x^{2}, x^{3}$, where we assume the Minkowski metric is $d s^{2}=\left(d x^{0}\right)^{2}-\left(d x^{1}\right)^{2}-\left(d x^{2}\right)^{2}-\left(d x^{3}\right)^{2}$.

The metric $d s^{2}$ on $M_{0}$ induces a Hodge *-operator.

$$
\text { *: } \bigwedge^{p} T^{*}\left(M_{0}\right) \rightarrow \bigwedge^{4-p} T^{*}\left(M_{0}\right)
$$

We recall (cf. de Rham [6]) that if

$$
\alpha=\alpha_{i_{1} \ldots i_{p}} d x^{i_{1}} \wedge \cdots \wedge d x^{i_{p}}
$$

then

$$
(* \alpha)_{j_{1}, \ldots, j_{4-p}}= \pm \alpha^{i_{1} \ldots i_{p}}
$$

where $\left\{i_{1}, \ldots, i_{p}, j_{1}, \ldots, j_{4-p}\right\}$ is an odd or even permutation of $\{0,1,2,3\}$, which determines the above sign, and

$$
\alpha^{i_{1} \ldots i_{p}}=g^{i_{1} k_{1}} g^{i_{2} k_{2}} \ldots g^{i_{p} k_{p}} \alpha_{k_{1}} \ldots k_{p}
$$

where

$$
\left[g^{i j}\right]=\left[\begin{array}{rrrr}
+1 & 0 & 0 & \\
0 & -1 & 0 & 0 \\
0 & 0 & -1 & 0 \\
0 & 0 & 0 & -1
\end{array}\right]
$$

This introduces some minus signs into the usual Euclidean $*$-formalism (cf. Wells [32]). In this case we find that

$$
* *(p \text {-form })=(-1)^{p+1}(p \text {-form }),
$$

so $* 2=-1$, when acting on 2 -forms in $M_{0}$. Therefore $*$ has eigenvalues $\pm i$ in this case. Considering $C$-valued 2-forms on $M_{0}$ we have

$$
\wedge^{2} T^{*}\left(M_{0}\right) \otimes \mathrm{C}=\wedge_{+}^{2}\left(M_{0}\right) \oplus \bigwedge_{-}^{2}\left(M_{0}\right),
$$

where $\wedge_{+}^{2}$ and $\Lambda_{-}^{2}$ denote the $+i$ and $-i$ eigenspaces. So any 2-form $w \in \mathcal{E}^{2}\left(M_{0}\right)$ has a decomposition $w=w^{+}+w^{-}$, where

$$
w^{+}=\frac{1}{2}(w-i * w), \quad w^{-}=\frac{1}{2}(w+i * w),
$$

satisfy $* w^{+}=i w, * w^{-}=-i w$. We say that $w$ is a self-dual if $w=w^{+}$and anti-self-dual if $w=w^{-}$. We now have the following two propositions. 
7.1 Proposition. (1) Maxwell's (homogeneous) equations become

$$
d F=0, d^{*} F=0 \text {. }
$$

(2) If the Maxwell tensor is rewritten as $F=F^{+}+F^{-}$, then Maxwell's equations become

$$
d F^{+}=d F^{-}=0 .
$$

The proof of this proposition is simply a translation of the notation. $d^{*}$ is the Hodge adjoint to $d$ and is $= \pm * d *$.

We now want to switch to use spinor coordinates in $M_{0}$.

$$
x^{a} \rightarrow x^{A A^{\prime}}=\frac{1}{\sqrt{2}}\left[\begin{array}{ll}
x^{0}+x^{1} & x^{2}+i x^{3} \\
x^{2}-i x^{3} & x^{0}-x^{1}
\end{array}\right]
$$

as in $\S 6$. We want to rewrite $F=F_{a b}$, the Maxwell tensor, in terms of spinors, and express $F^{+}$and $F^{-}$in terms of spinors also.

We have

$$
F_{a b} \sim F_{A A^{\prime} B B^{\prime}}, \quad \text { where } F_{A A^{\prime} B B^{\prime}}=-F_{B B^{\prime} A A^{\prime}}
$$

and where we associate $F_{a b}$ with its image under the injection $\mathscr{T}\left(M_{0}\right) \rightarrow M_{0} \times$ T(S). So

$$
\begin{aligned}
F_{A A^{\prime} B B^{\prime}} & =\frac{1}{2}\left(F_{A A^{\prime} B B^{\prime}}-F_{B B^{\prime} A A^{\prime}}\right) \\
& =\frac{1}{2}\left(F_{A A^{\prime} B B^{\prime}}-F_{B A^{\prime} A B^{\prime}}+F_{B A^{\prime} A B^{\prime}}-F_{B B^{\prime} A A^{\prime}}\right) \\
& =\frac{1}{2}\left(\varepsilon_{A B} F_{M A^{\prime}}{ }^{M}{ }_{B^{\prime}}+\varepsilon_{A^{\prime} B^{\prime}} F_{B M^{\prime} A^{\prime}}{ }^{M^{\prime}}\right)
\end{aligned}
$$

which is easy to check. Now let

$$
\varphi_{A B}=\frac{1}{2} F_{B M^{\prime} A^{\prime}} M^{\prime}, \quad \psi_{A^{\prime} B^{\prime}}=\frac{1}{2} F_{M A^{\prime}}{ }^{M}{ }_{B^{\prime}}
$$

Then

$$
\varphi_{A B}=\varphi_{B A}, \quad \psi_{A^{\prime} B^{\prime}}=\psi_{B^{\prime} A^{\prime}},
$$

and moreover

$$
F_{a b} \sim F_{A A^{\prime} B B^{\prime}}=\varepsilon_{A B} \psi_{A^{\prime} B^{\prime}}+\varepsilon_{A^{\prime} B^{\prime}} \varphi_{A B} .
$$

7.3 Proposition. (1) $F$ is real if and only if $\psi_{A^{\prime} B^{\prime}}=\overline{\varphi_{A B}}$.

(2) $F$ is self-dual (i.e. $* F=i F)$ if and only if $F=\varepsilon_{A B} \psi_{A^{\prime} B^{\prime}}$

(3) $F$ is anti-self-dual (i.e. $* F=-i F$ ) if and only if $F=\varepsilon_{A^{\prime} B^{\prime}} \varphi_{A B}$.

The proof is computational, and will be omitted (cf. Hanson and Newman [9]); it depends on the following formula for the *-operator [28].

$$
(* F)_{A A^{\prime} B B^{\prime}}=i\left(\varepsilon_{A B} \psi_{A^{\prime} B^{\prime}}-\varepsilon_{A^{\prime} B^{\prime}} \varphi_{A B}\right) \text {. }
$$

The representation (7.2) splits $F_{a b} \sim F_{A A^{\prime} B B^{\prime}}$ into the sum of two quantities $\varepsilon_{A B} \psi_{A^{\prime} B^{\prime}}$, where $\varepsilon_{A B}$ is a constant matrix $\left[\begin{array}{cc}0 \\ -1\end{array}\right]$ and $\psi_{A^{\prime} B^{\prime}}$ is a variable symmetric spinor field. The product $\varepsilon_{A B} \psi_{A^{\prime} B^{\prime}}$ is self-dual and corresponds to $F^{+}$in the preceding discussion. Similarly $\varepsilon_{A^{\prime} B^{\prime}} \varphi_{A B}$ is of the same nature. So the 6 independent coefficients of $F=F_{a b}$ is replaced by the 6 independent spinor quantities $\varphi_{00}, \varphi_{10}=\varphi_{01}, \varphi_{11}, \psi_{0^{\prime} 0^{\prime}}, \psi_{0^{\prime} 1^{\prime}}=\psi_{1^{\prime} 0^{\prime}}$ and $\psi_{1^{\prime} 1^{\prime}}$, and the relation between $\left\{\varphi_{A B}, \psi_{A^{\prime} B^{\prime}}\right\},\left\{F_{a b}\right\}$, and $\left\{E_{i}, B_{i}\right\}$ is a purely linear algebraic one. 
Now we want to reformulate Maxwell's equations using the spinor representation of a 2-form. Let $\left\{x^{A A^{\prime}}\right\}$ be the spinor coordinates of $M_{0}$, and define

$$
\nabla_{A A^{\prime}}=\frac{\partial}{\partial x^{A A^{\prime}}}, \quad \nabla^{A A^{\prime}}=\frac{\partial}{\partial x_{A A^{\prime}}},
$$

where $x_{A A}$ are dual variables expressed by

$$
x_{A A^{\prime}}=x^{B B^{\prime}} \varepsilon_{B A^{\prime}} \varepsilon_{B^{\prime} A^{\prime}}
$$

By the chain rule we compute easily that

$$
\nabla^{A A^{\prime}}=\varepsilon^{B A} \varepsilon^{B^{\prime} A^{\prime}} \nabla_{B B^{\prime}}
$$

7.4 Proposition. If

$$
F=F_{a b} d x^{a} \wedge d x^{b} \sim \varepsilon_{A B} \psi_{A^{\prime} B^{\prime}}+\varepsilon_{A^{\prime} B^{\prime}} \varphi_{A B},
$$

then Maxwell's equations for $F$ take the form

$$
\begin{array}{ll}
\nabla^{A A^{\prime}} \varphi_{A B}=0, & \varphi_{A B} \text { symmetric, } \\
\nabla^{A A^{\prime}} \psi_{A^{\prime} B^{\prime}}=0 & \psi_{A^{\prime} B^{\prime}} \text { symmetric. }
\end{array}
$$

Again we will not carry out the computations involved. But we note that there are 6 independent quantities $\left\{\varphi_{A B}\right\},\left\{\psi_{A^{\prime} B^{\prime}}\right\}$ as observed before, and there are 8 independent equations, just as at the beginning of this section, (recalling we are considering complex-valued solutions), e.g.

$$
\begin{aligned}
& \nabla^{00}{ }^{\prime} \varphi_{00}+\nabla^{10^{\prime}} \varphi_{10}=0, \\
& \nabla^{00} \varphi_{01}^{\prime}+\nabla^{11^{\prime}} \varphi_{11}=0, \\
& \nabla^{01^{\prime}} \varphi_{00}=\nabla^{11^{\prime}} \varphi_{10}=0, \text { etc. }
\end{aligned}
$$

These equations correspond to linear algebraic changes of variables in the variable and range space of the vector valued function defined on $M_{0}$ (components of $F$ ). In this form there is a generalization of these equations which one can easily write down.

Consider the differential equation on real or complexified Minkowski space (affine):

$$
\nabla^{A A^{\prime}} \varphi_{A B C \ldots D}=0, \quad \nabla^{A A^{\prime}} \psi_{A^{\prime} B^{\prime} \ldots D^{\prime}}=0,
$$

$\varphi_{A B C \ldots D}, \psi_{A^{\prime} B^{\prime} \ldots D^{\prime}}$ symmetric spinors with $2 s$ indices, $s=\frac{1}{2}, 1, \frac{3}{2}, \ldots$

These are the zero-rest-mass field equations (cf. Penrose [19], [20]). For $s=\frac{1}{2}$ : solutions correspond to neutrinos, this is the Dirac-Weyl "equation of an electron" for mass zero.

$s=1$ : solutions correspond to photons, these are Maxwell's equations above.

$s=2$ : solutions correspond to "weak gravitational fields;" these are the linearized Einstein equations, i.e. equations for a Lorentzian metric $h$ where $g=\eta+\varepsilon h, g$ a space-time metric, $h$ a perturbation, and $\eta$-the metric for flat space-time (due to Pauli-Fierz, cf. Penrose-MacCallum [25]).

In the next several sections we will discuss holomorphic representations of solutions of these equations. 
8. Cohomology and holomorphic vector bundles. In the classical theory of complex variables the basic object of study is the set of holomorphic functions on a domain $D \subset \mathbf{C}^{n}$. In modern complex analysis it has proven to be very useful to generalize these notions in various ways. First the domain $D$ in $\mathbf{C}^{n}$ is generalized to a complex manifold, a paracompact topological space $X$ which is locally homeomorphic to a domain in $\mathbf{C}^{n}$, and such that the overlap transition functions are holomorphic vector-valued functions. Basic examples of complex manifolds include $\mathbf{P}_{n}=\mathbf{P}_{n}(\mathbf{C})$ and the flag manifolds discussed in §4. These are compact complex manifolds. The open subset $\mathbf{P}_{3}^{+}$of $\mathbf{P}_{3}$ described in $\$ 4$ is an example of a noncompact complex manifold which is not biholomorphic to an open subset of $\mathbf{C}^{n}$, since one can show that there are no nonconstant holomorphic functions on $\mathbf{P}_{3}^{+}$(this is due to the proliferation of compact complex submanifolds of $\mathbf{P}_{3}^{+}$of complex dimension 1, cf. Proposition 4.6). The second generalization we consider is from a (continuous, $C^{\infty}$, or holomorphic) vector-valued function defined on a complex manifold $X$ to a section of a holomorphic vector bundle over $X$. Basically, a holomorphic vector bundle over a complex manifold $X$ with fiber $\mathbf{C}^{r}$ is a mapping $V \stackrel{\pi}{\rightarrow} X$ where $V$ and $X$ are complex manifolds and $\pi$ is a surjective holomorphic mapping of maximal rank which satisfies: (a) for each $p \in X, \pi^{-1}(p)$ is a C-linear vector space of dimension $r$, the fiber over $p$, and (b) for each $p \in X$, there is a neighborhood $U$ of $p$ and a fiber-preserving biholomorphic equivalence $\pi^{-1}(U) \stackrel{\cong}{\cong} U \times \mathbf{C}^{r}$, which is $\mathbf{C}$-linear on the fibers. A section of $V$ over $X$ is a mapping $s: X \rightarrow V$ such that $\pi \circ s=\mathrm{id}_{X}$, i.e., to each point $p \in X, s(x)$ is a point in the fiber $V_{p}=\pi^{-1}(p)$ over $p$. Then locally, near $p \in X, s(q)$ is a vector in $\mathbf{C}^{r}$ using the (nonunique) local product representation. In other words a section of a holomorphic vector bundle is locally representable (or identifiable with) a $\mathbf{C}^{r}$-valued function.

Holomorphic vector bundles and their sections are important generalizations of holomorphic functions in several variables and these have various applications in problems concerning complex manifolds which are not a subdomain of $\mathbf{C}^{n}$. For example some open subsets of $\mathbf{P}_{n}\left(\right.$ e.g., $\mathbf{P}_{3}^{+}$, for $n=3$ ), will have nontrivial vector bundles on them. Examples include the tangent bundle to a complex manifold and various bundles generated by linear algebraic operations on the fibers of these bundles, e.g., $\oplus, \otimes$, duality, etc.

Let us give one example which will be important for our purposes in this paper. First we give a little bit of notation which helps describe bundles. If $X$ is a complex manifold and $V \stackrel{\pi}{\rightarrow} X$ is a holomorphic vector bundle over $X$, then there is a covering $\left\{U_{\alpha}\right\}$ of $X$ and local trivializations (see (b) above) $\varphi_{\alpha}$ : $\pi^{-1}\left(U_{\alpha}\right) \rightarrow U_{\alpha} \times \mathbf{C}^{\gamma}$ which induce transition functions $g_{\alpha \beta}=\varphi_{\alpha} \circ \varphi_{\beta}^{-1}$ which map holomorphically $U_{\alpha} \cap U_{\beta} \rightarrow G L(n, \mathbf{C})$ and satisfy the cocycle conditions $g_{\alpha \beta} \cdot g_{\beta \gamma} \cdot g_{\gamma \alpha}=i d$. It is easy to see that the transition functions $\left\{g_{\alpha \beta}\right\}$ determine the equivalence class of a given bundle, and that a set of transition functions satisfying the cocycle condition will determine (an equivalence class of) a holomorphic vector bundle. Now consider $\mathbf{P}_{n}$, and letting $\left[z^{0}\right.$, $\left.z^{1}, \ldots, z^{n}\right]$ be homogeneous coordinates for a point in $\mathbf{P}_{n}$, set $U_{\alpha}=$ $\left\{\left[z^{0}, z^{1}, \ldots, z^{n}\right]: z^{\alpha} \neq 0\right\}$. Define

$$
g_{\alpha \beta}\left(\left[z^{0}, \ldots, z^{n}\right]\right)=z^{\beta} / z^{\alpha} \text { on } U_{\alpha} \cap U_{\beta},
$$


then

$$
g_{\alpha \beta}: U_{\alpha} \cap U_{\beta} \rightarrow G L(1, \mathbf{C}) \cong \mathbf{C}-\{0\},
$$

and $\left\{g_{\alpha \beta}\right\}$ satisfy the cocycle condition. This defines a holomorphic line bundle on $\mathbf{P}_{n}$ (a holomorphic vector bundle with fiber $\mathbf{C}$ ); it is called the hyperplane section bundle, denoted by $H \rightarrow \mathbf{P}_{n}$. We define $H^{m} \rightarrow \mathbf{P}_{n}$ by the powers of the transition functions $\left\{g_{\alpha \beta}^{m}\right\}$. It is a well understood fact that the naturally defined holomorphic equivalence classes of holomorphic line bundles on $\mathbf{P}_{n}$ is isomorphic to the discrete set of bundles $\left\{H^{m}\right\}_{m \in \mathbf{Z}}$. Here $H^{0}$ is a trivial line bundle $\cong \mathbf{P}_{n} \times \mathbf{C}$.

On a complex manifold $X$ there are $C^{\infty}$ differential forms of type $(p, q)$ described locally by

$$
\varphi=\sum_{\substack{i_{1}<\ldots<i_{p} \\ j_{1}<\cdots<j_{q}}} \varphi_{i_{1} \ldots i_{j} j_{1} \ldots j_{q}} d z^{i_{1}} \wedge \cdots \wedge d z^{i_{p}} \wedge d \bar{z}^{j_{1}} \wedge \cdots \wedge d \bar{z}^{j_{q}},
$$

where $\varphi_{i_{1}} \ldots i_{j} j_{1} \ldots j_{q}$ are $C^{\infty}$ functions, and $d z^{j}=d x^{j}+i d y^{j}, d \bar{z}^{j}=d x^{j}-$ $i d y^{j}, j=1, \ldots, n$, are the locally defined 1 -forms coming from the complex structure, where $z_{j}=x_{j}+i y_{j}, j=1, \ldots, n$, are local holomorphic coordinates on $X$.

If $\mathcal{E}^{p, q}(X)$ denotes the differential forms of type $(p, q)$ on $X$, and $\mathcal{E}^{r}(X)$ denotes the (complex-valued) differential forms of degree $r$ on $X$, then there is a natural projection $\pi_{p, q}: \mathcal{E}^{p+q}(X) \rightarrow \mathcal{E}^{p, q}(X)$. This induces a differential operator complex

$$
\rightarrow \mathcal{E}^{p, q-1}(X) \stackrel{\bar{\partial}}{\rightarrow} \mathcal{E}^{p, q}(X) \stackrel{\bar{\partial}}{\rightarrow} \mathcal{E}^{p, q+1}(X) \rightarrow
$$

defined by $\bar{\partial} \varphi=\left(\pi_{p, q} \circ d\right) \varphi$, where $d$ is the usual exterior derivative of the differential form $\varphi$. The holomorphic functions on $X$ are denoted by $\theta(X)$ and are the kernel of the mapping $\bar{\partial}$ acting on functions (differential forms of type $(0,0))$, i.e.,

$$
0 \rightarrow \vartheta(X) \rightarrow \mathcal{E}^{0,0}(X) \stackrel{\hat{\jmath}}{\rightarrow} \mathcal{E}^{0,1}(X)
$$

is exact. Obstruction to $(8.1)$ being exact for $(p, q) \neq(0,0)$ is part of the cohomology we will meet below.

Now that we have the concept of a holomorphic section of a holomorphic vector bundle $V \rightarrow X$, and the notion of a differential form of type $(p, q)$ on $X$, we put these two concepts together in defining a differential form with coefficients in a holomorphic vector bundle. First a section of a holomorphic vector bundle $V$ is given by locally defined vector-valued functions which satisfy compatibility conditions given by the transition functions for the given bundle. So if $V \stackrel{\pi}{\rightarrow} X$ is a holomorphic vector described by a covering $\left\{U_{\alpha}\right\}$ and transition functions $\left\{g_{\alpha \beta}\right\}$, then a section is a family of mappings $\left(C^{\infty}\right.$ or holomorphic, say) $S_{\alpha}: U_{\alpha} \rightarrow \mathbf{C}^{n}$ satisfying $S_{\alpha}(x)=g_{\alpha \beta}(x) S_{\beta}(x)$. A differential form-valued section (or equivalently a differential form with coefficients in a holomorphic vector bundle) is a collection of vector-valued differential forms

$$
f_{\alpha} \in \mathcal{E}^{p, q}\left(U_{\alpha}\right) \otimes \mathbf{C}^{r}
$$


satisfying

$$
f_{\alpha}(x)=g_{\alpha \beta}(x) f_{\beta}(x), \quad x \in U_{\alpha} \cap U_{\beta},
$$

where the matrix $g_{\alpha \beta}(x)$ acts on the vector of differential forms by the usual matrix multiplication. We denote the vector space of all such differential forms of type $(p, q)$ with coefficients in $V$ by $\mathcal{E}^{p, q}(X, V)$, and the $\bar{\partial}$-operator extends naturally to these more general forms, i.e., if $f=\left\{f_{\alpha}\right\}$, then define $\bar{\partial} f=\left\{\bar{\partial} f_{\alpha}\right\}$. This is well defined, since $\bar{\partial}$ annihilates $g_{\alpha \beta}$ the holomorphic transition functions. Thus we obtain the general Dolbeault complex

$$
\ldots \rightarrow \mathcal{E}^{p, q-1}(X, V) \stackrel{\bar{\partial}}{\rightarrow} \mathcal{E}^{p, q}(X, V) \stackrel{\bar{\partial}}{\rightarrow} \mathcal{\delta}^{p, q+1}(X, V) \rightarrow \ldots
$$

satisfying $\bar{\partial}^{2}=0$. If we define

$$
H^{p, q}(X, V)=\frac{\operatorname{Ker} \bar{\partial}: \mathcal{E}^{p, q}(X, V) \rightarrow \mathcal{E}^{p, q+1}(X, V)}{\operatorname{Im} \bar{\partial}: \mathcal{E}^{p, q-1}(X, V) \rightarrow \mathcal{E}^{p, q}(X, V)},
$$

then we say that $H^{p, q}(X, V)$ is the cohomology group of type $(p, q)$ with coefficients in the holomorphic vector bundle $V$. If $V=X \times \mathbf{C}$ is the trivial line bundle then we write $H^{p, q}(X, V)=H^{p, q}(X)$, the cohomology of the original Dolbeault complex (8.1). There are various important alternative characterizations of $H^{p, q}(X, V)$ in terms of sheaf theory, Cech theory, etc., and in a given geometric or analytical setting one must choose the representation of this cohomology appropriately in order to be able to make computations. For the expository purposes in this paper, the above definition will suffice, but proofs of various assertions will of ten depend on understanding the alternative approaches to complex manifold cohomology available in the standard literature of the subject.

The cohomology groups $H^{p, q}(X, V)$ describe on $X$ holomorphic objects which are a generalization of $H^{0,0}(X, V)$, the vector space of holomorphic sections of $V$ over $X$, which was itself a generalization of ordinary vectorvalued holomorphic functions on $X$. The analogous quotient space of differential forms of total degree $r$ gives the de Rham group of $X$, i.e.,

$$
H^{r}(X)=\frac{(d \text {-closed } r \text {-forms })}{(d \text {-exact } r \text {-forms })},
$$

and its dimension is a topological invariant of $X$, the rth Betti number, a partial description of the global topological behavior of $X$. Similarly, $H^{p, q}(X, V)$ is a description of the global complex-analytic nature of a given complex manifold $X$ equipped with a vector bundle $V \rightarrow X$. When $H^{p, q}(X, V)$ vanishes for suitable $V$ and $(p, q)$, then this implies that obstructions to solving certain global complex-analytic problems vanish (e.g., the problem of Mittag-Leffler and Weierstrass in classical complex analysis can be formulated in this manner, or the existence of nontrivial global meromorphic functions with specified types of poles on a Riemann surface, the Riemann-Roch problem, is of this nature). If $H^{p, q}(X, V)$ is not zero, then the behavior of this vector space parallels in many ways the behavior of vector spaces of holomorphic or meromorphic functions on a given domain in 
$\mathbf{C}^{n}$ or $\mathbf{P}_{n}$. Cohomology classes in $H^{p, q}(X, V)$ can be considered as holomorphic objects which can be studied in the same manner that one might study holomorphic or meromorphic functions (cf. e.g., recent work in group representation theory and automorphic function theory; see Wells-Wolf [33] for references to this). These are the kinds of holomorphic objects which occur in Penrose's holomorphic representation of the solutions of the zerorest-mass field equations as described in the next section. For more background and details about the material discussed so briefly here the reader can consult Gunning and Rossi [8], Hirzebruch [11], Morrow and Kodaira [16], or Wells [32].

9. Holomorphic representation of solutions of the zero-rest-mass field equations. Let $H \rightarrow \mathbf{P}_{3}=\mathbf{P}_{3}(\mathbf{C})$ be the hyperplane section bundle described in $\S 8$, and consider $H^{p, q}\left(\mathbf{P}_{3}, H^{n}\right)$, the cohomology groups of $\mathbf{P}_{3}$ with coefficients in $H^{n}$. It is easy to verify that

$$
\begin{gathered}
H^{0,0}\left(\mathbf{P}_{3}, H^{n}\right)=\left\{\text { holomorphic polynomials in } \mathbf{C}^{4},\right. \\
\text { homogeneous of degree } n, n \geqslant 0 .\} \\
H^{0.0}\left(\mathbf{P}_{3}, H^{n}\right)=0, \quad n<0 .
\end{gathered}
$$

However, if $U$ is open in $\mathbf{P}_{3}$, we may have nontrivial sections of $H^{n}$, for $n<0$. For instance,

$$
f\left(Z^{0}, Z^{1}, Z^{2}, Z^{3}\right)=1 /\left(Z^{0}\right)^{3}
$$

defines a holomorphic section of $\mathrm{H}^{-3}$ for an appropriate open set which avoids the pole $Z^{0}=0$.

We want to consider $H^{0,1}\left(\mathbf{P}_{3}^{+}, H^{-2 s-2}\right), s=\frac{1}{2}, 1, \frac{3}{2}, \ldots$, and show how to obtain holomorphic solutions of the zero-rest-mass field equation of spin $s$ (7.6) in $M_{\mathrm{C}}^{+}$. These solutions will have real-analytic boundary values (satisfying the field equations) in $M_{0}$ if the cohomology class considered in $H^{0,1}\left(\mathbf{P}_{3}^{+}, H^{-2 s-2}\right)$ is actually a class in $H^{0,1}\left(\overline{\mathbf{P}_{3}^{+}}, H^{-2 s-2}\right)$. More generally we could consider hyperfunction boundary values in $M_{0}$ of holomorphic solutions in $M_{\mathbf{C}}^{+}$, but we won't get into this question in this paper.

If $V$ is a holomorphic vector bundle over a complex manifold $X$, we will set $\theta(X, V)=H^{0,0}(X, V)$, the global holomorphic sections of $V$ over $X$, and we will set $\Omega^{p}(X, V)=H^{p, 0}(X, V)$, the holomorphic $p$-forms with coefficients in $V$ on $X$, and $\Omega^{p}(X)$ denotes ordinary holomorphic $p$ forms, i.e., $H^{p, 0}(X)$.

There are two ways of representing cohomology which yields explicit solutions to the field equations: Čech coverings and Dolbeault forms with bundle coefficients. In terms of a Čech covering $\left\{U_{\alpha}\right\}$ on $\mathbf{P}_{3}^{+}$, with holomorphic sections $f_{\alpha \beta} \in \mathcal{O}\left(U_{\alpha} \cap U_{\beta}, H^{-2 s-2}\right)$, one can define certain contour integrals of the $f_{\alpha \beta}$ over contours contained in $U_{\alpha} \cap U_{\beta}$ to obtain spinor fields in $M_{\mathbf{C}}^{+}$satisfying the field equation (7.6). Basically each contour is in the projective complex line $\tau_{+}^{-1}\left(z^{A A^{\prime}}\right)$, where $z^{A A^{\prime}} \in M_{\mathrm{C}}^{+}$, and the contour integral is independent of the particular contour (but does depend on a certain homology class). Originally these contour integrals were of homogeneous holomorphic functions defined on certain open sets. It was only later that it was discovered that the integrals depended on the cohomology class 
defined by the given data. See Penrose [19], [20], and [23], as well as the very comprehensive article Penrose-MacCallum [25] which has many complex examples of such integrals worked out in detail. Once it was known that the cohomology class was the object of interest, one could find other means of mapping a cohomology class to a solution of the field equation, e.g. the "Sparling-Ward splitting method" (cf. [29]), and Woodhouse's Dolbeault representation [35].

We will describe only one of three methods, namely the one due to Woodhouse [35]. We'll write down a specific integral formula involving differential forms and show that it satisfies the field equations (7.6). In the following section we will briefly discuss how to interpret this representation in a coordinate free manner. First we will introduce some useful notation, and discuss briefly homogeneous differential forms on $\mathbf{C}^{4}$.

Suppose we have twistor homogeneous coordinates $Z=\left(Z^{0}, Z^{1}, Z^{2}, Z^{3}\right)$, in which $\Phi(Z)=Z^{0} \bar{Z}^{2}+Z^{1} \bar{Z}^{3}+Z^{2} \bar{Z}^{0}+Z^{3} \bar{Z}^{1}$. We will denote these coordinates by $Z^{\alpha}(\alpha=0,1,2,3)$, similar to our notation for coordinates in Minkowski space $M_{\mathrm{C}}^{A}, z^{A A^{\prime}}$ (cf. §6). We define the dual variables with respect to the Hermitian form $\Phi$ by

$$
\bar{Z}_{\alpha}: \bar{Z}_{0}=\overline{Z^{2}}, \quad \bar{Z}_{1}=\overline{Z^{3}}, \quad \bar{Z}_{2}=\overline{Z^{0}}, \quad \bar{Z}_{3}=\overline{Z^{1}},
$$

and thus $\Phi\left(Z^{\alpha}\right)=Z^{\alpha} \bar{Z}_{\alpha}$. This is the notation used in the papers of Penrose. Define

$$
\omega^{A}=\left(\omega^{0}, \omega^{1}\right):=\left(Z^{0}, Z^{1}\right) \quad \pi_{A^{\prime}}=\left(\pi_{0}, \pi_{1^{\prime}}\right):=\left(Z^{2}, Z^{3}\right),
$$

so that the 4-tuple of complex numbers $Z^{\alpha}=\left(Z^{0}, Z^{1}, Z^{2}, Z^{3}\right)$ becomes the pair of spinors $\left(\omega^{A}, \pi_{A^{\prime}}\right)$. The dual coordinates become:

$$
\bar{Z}_{\alpha}=\left(\bar{\pi}_{A}, \bar{\omega}^{A^{\prime}}\right) \text {, }
$$

and

$$
\varphi\left(Z^{\alpha}\right)=\omega^{A} \bar{\pi}_{A}+\pi_{A^{\prime}} \bar{\omega}^{A^{\prime}}
$$

REMARK. If a particle of zero-rest-mass moves along a light ray then $\pi^{A} \bar{\pi}^{A^{\prime}}$ corresponds to the momentum and $\omega^{A} \bar{\omega}^{A^{\prime}}$ corresponds to the angular momentum, and the form $\Phi\left(Z^{\alpha}\right)$ given above corresponds to the spin (or twist) of the particle. The path of motion of the particle is given by $\tau\left(\left[\omega^{A}, \pi_{A^{\prime}}\right]\right)$. See Penrose-MacCallum [25] for a further discussion of this interpretation. We note further that the action of $S L(2, \mathrm{C})$ on spinors preserves $(9.1)$, and thus it is natural to consider $S U(2,2)$ the full group of transformations which preserve (9.1), which is what we did in $\$ 4$.

Thus $\left(\omega^{A}, \pi_{A^{\prime}}\right)$ corresponds to $\mathbf{C}^{4}=\mathbf{C}^{2} \oplus \mathbf{C}^{2}$, with $\omega^{A}$ as coordinates on the first summand and $\pi_{A^{\prime}}$ as coordinates on the second summand. These coordinates will be used in our integral formula.

We now turn to homogeneous differential forms on $\mathbf{C}^{4}$ (we could just as well study $\mathbf{C}^{n}$, but for continuity we'll stick to $\mathbf{C}^{4}$ ). Let $Y=Z^{\alpha} \partial / \partial Z^{\alpha}$ be the Euler vector field on $\mathbf{C}^{4} . Y$ and $\bar{Y}$ are tangent to the fibers of the projection $\mathbf{C}^{4}-\{0\} \stackrel{\pi}{\rightarrow} \mathbf{P}_{3}$. Suppose $U \subset \mathbf{P}_{3}$ is open, then we want to describe $\pi^{*}\left(\mathscr{E}^{p}(U)\right) \subset \mathscr{E}^{p}(\hat{U})$, where $\hat{U}=\pi^{-1}(U)$. Essentially we need the form to be constant along the fibers; and the following lemma is not difficult to verify. 
9.2 Lemma. $\left.\left.\pi^{*}: \mathcal{E}^{p}(U) \rightarrow\left\{f \in \mathcal{E}^{p}(\hat{U}): \quad Y\right\lrcorner f=0, \quad \bar{Y}\right\lrcorner f=0, \quad Y\right\lrcorner d f=0$, $\bar{Y}\lrcorner d f=0\}$ is an isomorphism.

Here $Y\lrcorner f$ means interior product of a vector field and a differential form. We see that if $p=0$, the first conditions are vacuous, and the second conditions are just $Y(f)=\bar{Y}(f)=0$, i.e. $f$ is constant on the fibers of $\pi$ (homogeneous of degree 0 ). The conditions in Lemma 8.2 say that the restriction of $f$ and $d f$ as a differential form to the fibers of $\pi$ vanish identically.

We want to consider differential forms with coefficients in $H^{n}$. Consider first holomorphic forms, and we have, just as in Lemma 9.2, if $U$ is open in $\mathbf{P}_{3}$, and $\hat{U}=\pi^{-1}(U)$,

$$
\left.\left.\Omega^{1}(U) \cong\left\{f \in \Omega^{p}(\hat{U}): Y\right\lrcorner f=0, Y\right\lrcorner \partial f=0\right\},
$$

(since $\bar{Y}\lrcorner f=\bar{Y}\lrcorner d f=0$ automatically). Now consider

$$
\left.\Omega^{0}\left(U, H^{m}\right)=\mathcal{O}\left(U, H^{m}\right) \cong\{f \in \mathcal{O}(\hat{U}): Y\lrcorner \partial f=m f\right\},
$$

and we have the natural generalization of this to holomorphic $p$-forms

$$
\left.\left.\Omega^{p}\left(U, H^{m}\right) \cong\left\{f \in \Omega^{p}(\hat{U}): Y\right\lrcorner f=0, Y\right\lrcorner \partial f=m f\right\} .
$$

We will give an example of this which will be useful to us later. Define

$$
\theta=I_{\alpha \beta} Z^{\alpha} d Z^{\beta} \text {, }
$$

where $I_{\alpha \beta}$ is a $4 \times 4$ matrix defined by

$$
I=\left[\begin{array}{ll}
0 & 0 \\
0 & \varepsilon^{A^{\prime} B^{\prime}}
\end{array}\right]
$$

It is easy to compute that $\theta$ depends only on the last two coordinates of $\mathbf{C}^{4}$, recalling that $Z^{\alpha}=\left(Z^{0}, Z^{1}, Z^{2}, Z^{3}\right)=\left(\omega^{A}, \pi_{A^{\prime}}\right)$, namely,

$$
\theta=-\pi^{E^{\prime}} d \pi_{E^{\prime}}=\pi_{E^{\prime}} d \pi^{E^{\prime}}
$$

Now

$$
Y=Z^{\alpha} \frac{\partial}{\partial Z^{\alpha}}=\omega^{\lambda} \frac{\partial}{\partial \omega^{A}}+\pi_{B^{\prime}} \frac{\partial}{\partial \pi_{B^{\prime}}}
$$

which implies that

$$
\begin{aligned}
Y\lrcorner \theta & \left.=\left(\omega^{A} \frac{\partial}{\partial \omega^{A}}+\pi_{B^{\prime}} \frac{\partial}{\partial \pi_{B^{\prime}}}\right)\right\lrcorner\left(-\pi^{E^{\prime}} d \pi_{E^{\prime}}\right) \\
& =\pi_{E^{\prime}} \pi^{E^{\prime}}=0 .
\end{aligned}
$$

Also,

$$
\begin{aligned}
Y\lrcorner \partial \theta & \left.=Z^{\gamma} \frac{\partial}{\partial Z^{\gamma}}\right\lrcorner I_{\alpha \beta} d Z^{\alpha} \wedge d Z^{\beta} \\
& \left.=Z^{\gamma} \frac{\partial}{\partial Z^{\gamma}}\right\lrcorner 2 \sum_{\alpha<\beta} I_{\alpha \beta} d Z^{\alpha} \wedge d Z^{\beta} \\
& =2 Z^{\gamma} I_{\alpha \beta} d Z^{\beta}=2 \theta .
\end{aligned}
$$

so $\theta$ is a representation in $T \cong \mathbf{C}^{4}$ of an element of $\Omega^{1}\left(\mathbf{P}_{3}, H^{2}\right)$. 
Consider now

$$
\psi=h \cdot f \wedge \theta
$$

where each component $h, f, \theta$ are defined on $\hat{U}=\pi^{-1}(U), U_{\text {open }} \subset \mathbf{P}_{3}$ and

$$
\begin{aligned}
h= & \text { homogeneous holomorphic polynomial } \\
& \text { of degree } n \text { in } \mathbf{C}^{4} . \\
f= & \bar{\partial} \text {-closed }(0,1) \text {-form satisfying } \\
& \bar{Y}\lrcorner f=0, Y\lrcorner \partial f=(-n-2) f . \\
\theta= & \text { holomorphic }(1,0) \text {-form satisfying } \\
& Y\lrcorner \theta, Y\lrcorner \partial \theta=2 \theta .
\end{aligned}
$$

Then $\psi$ is a well-defined $\bar{\partial}$-closed $(1,1)$-form on $\hat{U}$ satisfying the condition of Lemma 9.2, and is therefore the lift of a form from $U$. Namely,

$$
\begin{aligned}
Y\lrcorner \psi= & h \cdot f(Y\lrcorner \theta)=0, \\
\bar{Y}\lrcorner \psi= & h(\bar{Y}\lrcorner f) \cdot \theta=0, \\
Y\lrcorner d \psi= & Y\lrcorner(d h \wedge f \wedge \theta+h d f \wedge \theta+h f \wedge d \theta) \\
= & Y(h) \cdot f \wedge \theta+h(Y\lrcorner d f) \theta \\
& +h f \wedge(Y\lrcorner d \theta), \\
= & n h \cdot f \wedge \theta+(-n-2) f \wedge h \theta \\
& +2 h f \wedge \theta=0, \\
\bar{Y}\lrcorner d \psi= & h(\bar{Y}\lrcorner d f)=h(\bar{Y}\lrcorner \partial f) \theta \\
= & h(\partial(\bar{Y}\lrcorner f)) \theta=0 .
\end{aligned}
$$

So assume that $f \in H^{0,1}\left(P_{3}^{+}, H^{-n-2}\right)$, and that $f$ is represented in $T^{+}$by $f \in \mathcal{E}^{0,1}\left(T^{+}\right)$satisfying $\left.\left.\overline{\partial f}=0, \bar{Y}\right\lrcorner f=0, Y\right\lrcorner \partial f=(-n-2) f$. Suppose that $h \in \mathcal{O}\left(\mathbf{P}_{3}^{+}, H^{n}\right)$, and is represented by a holomorphic homogeneous polynomial in $T^{+}$of homogeneity $n$. Suppose that $h$ is spinor-valued, with values in $S_{1,0}^{*} \underset{(n \text {-factors })}{\otimes} \otimes S_{0,1}^{*}$, i.e.,

$$
h\left(Z^{\alpha}\right)=h_{A^{\prime} \ldots D^{\prime}}(Z) e^{A^{\prime}} \otimes \cdots \otimes e^{D^{\prime}},
$$

where $\left\{e^{A^{\prime}}\right\}$ is a basis for $S_{0,1}^{*}$, and $h_{A^{\prime} \ldots D^{\prime}}\left(Z^{\alpha}\right)$ is a homogeneous holomorphic polynomial of degree $n$ for each set of indices.

Then we can form

$$
h \cdot f \wedge \theta \in \mathcal{E}^{1,1}(\hat{U}) \otimes S_{0,1}^{*} \underset{(n \text {-factors })}{\otimes} S_{0,1}^{*} \cdot
$$

So if we write

$$
h_{A^{\prime} \ldots D^{\prime}}(Z) f(Z) \wedge \theta(Z)
$$

for the coefficients of this spinor-valued differential form, we see that we have simply a vector-valued 2 -form with coefficients in a trivial vector bundle

$$
\mathbf{P}_{3}^{+} \times S_{0,1}^{*} \otimes \cdots \otimes S_{0,1}^{*} \text {. }
$$


Now (9.4) above is a well-defined $d$-closed $(1,1)$-form on $\mathbf{P}_{3}^{+}$for each fixed set of indices $\left(A^{\prime}, \ldots, D^{\prime}\right)$. So we can integrate this over any compact 2-manifold in $\mathbf{P}_{3}^{+}$and get a number depending on the indices. The basic formula depends on the right choice of $h_{A^{\prime}} \ldots D^{\prime}, \theta$, and the set of submanifolds.

Let $\theta$ be defined by (9.3), let

$$
h_{A^{\prime} B^{\prime} \ldots D^{\prime}}(Z)=\pi_{A^{\prime}} \pi_{B^{\prime}} \underset{(n \text { factors })}{\ldots} \pi_{D^{\prime}}
$$

which is a homogeneous polynomial in the coordinates $\left(\omega^{A}, \pi_{A^{\prime}}\right)$ of $T$, depending on the spinor indices. Let $z^{A A^{\prime}}$ be a fixed point in $M_{\mathbf{C}}^{+}$, and define

$$
\varphi_{A^{\prime} \ldots D^{\prime}}\left(z^{A A^{\prime}}\right)=\int_{\tau^{-1}\left(z^{A A^{\prime}}\right)} \pi_{A^{\prime}} \pi_{B^{\prime}} \ldots \pi_{D^{\prime}} f \wedge \theta,
$$

where $f \in H^{0,1}\left(\mathbf{P}_{3}^{+}, H^{-n-2}\right)$. So this integral is well defined with spinor values, and moreover it is symmetric in the indices.

9.6 THEOREM. The function $\varphi_{A^{\prime}} \ldots D^{\prime}$ defined by (9.5) is holomorphic, symmetric in $A^{\prime} \ldots D^{\prime}$ and satisfies the zero-rest-mass field equations of spin $s=n / 2$,

$$
\nabla^{A A^{\prime}} \varphi_{A^{\prime} \ldots D^{\prime}}=\mathbf{0}
$$

on $M_{\mathbf{C}}^{+}$; moreover the integral in (8.5) depends only on the Dolbeault cohomology class in $\mathrm{H}^{0,1}\left(\mathbf{P}_{3}^{+}, \mathrm{H}^{-n-2}\right)$.

Proof. Let

$$
I_{A^{\prime} \ldots D^{\prime}}(Z)=\pi_{A^{\prime}} \cdots \pi_{D^{\prime}} f(Z) \wedge \theta(Z)
$$

be the integrand in (9.5) above, where we have $Z=\left(\omega^{A}, \pi_{A^{\prime}}\right)$ are coordinates, as before. Suppose $z^{A A^{\prime}} \in M_{\mathrm{C}}^{+}$, then

(9.7) the two-plane in $T$ parametrized under the Penrose correspondence by $z^{A A^{\prime}}$ is the set of all $\left(\omega^{A}, \pi_{A^{\prime}}\right) \in T$ such that

$$
\omega^{A}=i z^{A A^{\prime}} \pi_{A^{\prime}},
$$

i.e., the graph over the $\mathbf{C}^{2}$-plane parametrized by $\left\{\pi_{A^{\prime}}\right\}$ in $\mathbf{C}^{4}=\mathbf{C}^{2} \times \mathbf{C}^{2}$ of a $\mathbf{C}^{2}$-valued linear function.

We have the following diagram:

$$
\begin{array}{ccc}
\left(\pi_{A^{\prime}}, z^{A A^{\prime}}\right) & \stackrel{\alpha_{0}}{\mapsto} & \left\{\left(\omega^{A}, \pi_{A^{\prime}}\right): \omega^{A}=i z^{A A^{\prime}} \pi_{A^{\prime}}\right\} \\
\pitchfork & & m \\
\mathbf{C}^{2} \times M_{\mathbf{C}}^{+} & \stackrel{\alpha_{0}}{\rightarrow} & T^{+} \\
\downarrow & & \downarrow \\
F^{+}=\mathbf{P}_{1} \times M_{\mathbf{C}}^{+} & \stackrel{\alpha}{\rightarrow} & \mathbf{P}^{3} \\
\downarrow & & \\
M_{\mathbf{C}}^{+} & &
\end{array}
$$

Remark. In (9.8) $\mathbf{P}_{1} \times M_{\mathbf{C}}^{+}$is an explicit representation of the flag domain $F^{+}$, depending on the coordinatization $\left(\omega^{A}, \pi_{A^{\prime}}\right)$ of $T$, which was a coordinate system in which $\Phi$ had the form $\Phi_{2}$. These coordinates are not $S U(2,2)$ invariant, and $\mathbf{P}_{1} \times M_{\mathbf{C}}^{+}$is not an $S U(2,2)$ invariant representation of $F^{+}$. 
We have, in view of the above coordinates, $I_{A^{\prime} \ldots D^{\prime}}\left(Z^{\alpha}\right)=I_{A^{\prime} \ldots D^{\prime}}\left(\omega^{A}, \pi_{A^{\prime}}\right)$, a $(1,1)$-form which projects to a well-defined spinor-valued $(1,1)$-form on $\mathbf{P}_{3}^{+}$, and which is symmetric in the indices, which we denote also by $I_{A^{\prime}} \ldots D^{\prime}$. Thus we have the pullback $\alpha^{*}\left(I_{A^{\prime}} \ldots D^{\prime}\right)$ is a well-defined $(1,1)$-form on $F^{+}=\mathbf{P}_{1} \times$ $M_{\mathrm{C}}^{+}$. We can integrate over the fibers of the projection $\beta$ to obtain

$$
\varphi_{A^{\prime} \ldots D^{\prime}}\left(z^{A A^{\prime}}\right)=\int_{\mathbf{P}_{1} \times\left\{z^{A A^{\prime}}\right\}} \alpha_{0}^{*} I_{A^{\prime} \ldots D^{\prime}}\left(\pi_{A^{\prime}}, z^{A A^{\prime}}\right) .
$$

We see that $\varphi_{A^{\prime} \ldots D^{\prime}}$ is holomorphic in $M_{C}^{+}$by differentiating under the integral sign in the integral in (9.7), and we omit this computation, as it is similar to but easier than the next one. We want to verify that $\varphi_{A^{\prime}} \ldots D^{\prime}$ given by (9.9) does indeed satisfy the zero-rest-mass field equations, and to do that we also simply differentiate under the integral sign. Namely we have,

$$
\frac{\partial}{\partial z^{A A^{\prime}}} \varphi_{B^{\prime} \ldots D^{\prime}}\left(z^{A A^{\prime}}\right)=\int_{\mathbf{P}_{1}} \frac{\partial}{\partial z^{A A^{\prime}}}\left(\alpha_{0}^{*} I_{B^{\prime} \ldots D^{\prime}}\right)\left(\pi_{A^{\prime}}, z^{A A^{\prime}}\right) .
$$

We have to write out the integrand to compute the derivative, and we work in homogeneous coordinates. Namely, we have

$$
I_{B^{\prime} \ldots D^{\prime}}\left(\omega^{E}, \pi_{E^{\prime}}\right)=-\pi_{B^{\prime}} \ldots \pi_{D^{\prime}}\left(f^{\alpha} d \bar{Z}_{\alpha}\right) \wedge \pi^{E^{\prime}} d \pi_{E^{\prime}}
$$

Write

$$
f^{\alpha} d \bar{Z}_{\alpha}=f_{M^{\prime}} d \bar{\omega}^{M^{\prime}}+f^{M} d \bar{\pi}_{M}
$$

where

$$
f_{M^{\prime}}=f_{M^{\prime}}\left(\omega^{N}, \pi_{N^{\prime}}\right), \quad f^{M}=f^{M}\left(\omega^{N}, \pi_{N^{\prime}}\right)
$$

Then

$$
\alpha_{0}^{*} I_{B^{\prime}} \ldots D^{\prime}\left(\pi_{A^{\prime}}, z^{A A^{\prime}}\right)=-\pi_{B^{\prime}} \ldots \pi_{D^{\prime}}\left\{f_{M^{\prime}} d\left(i \bar{z}^{M^{\prime} M} \bar{\pi}_{M}\right)+f^{M} d \bar{\pi}_{M}\right\} \wedge \pi^{E^{\prime}} d \pi_{E^{\prime}}
$$

Differentiating we find that

$$
\begin{aligned}
& \frac{\partial}{\partial z^{A A^{\prime}}}\left(\alpha_{0}^{*} I_{B^{\prime}} \ldots D^{\prime}\right)\left(\pi_{A^{\prime}}, z^{A A^{\prime}}\right) \\
& =-\pi_{B^{\prime}} \ldots \pi_{D^{\prime}} \pi^{E^{\prime}} d \pi_{E^{\prime}} \wedge \frac{\partial}{\partial z^{A A^{\prime}}}\left\{f_{M^{\prime}} d\left(-i \bar{z}^{M^{\prime} M} \bar{\pi}_{M}\right)+f^{M} d \pi_{M}\right\} \\
& =-\pi_{B^{\prime}} \ldots \pi_{D^{\prime}} \pi^{E^{\prime}} d \pi_{E^{\prime}}\left\{\frac{\partial f_{M^{\prime}}}{\partial \omega^{A}} \frac{\partial \omega^{A}}{\partial z^{A A^{\prime}}} d \bar{\omega}^{M^{\prime}}+\frac{\partial f^{M}}{\partial \omega^{A}} \frac{\partial \omega^{A}}{\partial z^{A A^{\prime}}} d \bar{\pi}_{M}\right\}
\end{aligned}
$$

where

So we find that

$$
\left.d \bar{\omega}^{A^{\prime}}\right|_{\tau^{-1}\left(z^{A A^{\prime}}\right)}=-i \bar{z}^{M^{\prime} M} d \bar{\pi}_{M}
$$

$$
\begin{gathered}
\frac{\partial}{\partial z^{A A^{\prime}}}\left(\alpha_{0}^{*} I_{B^{\prime}} \ldots D^{\prime}\right)\left(\pi_{A^{\prime}}, z^{A A^{\prime}}\right) \\
=-\left(i \pi_{A^{\prime}}\right) \pi_{B^{\prime}} \ldots \pi_{D^{\prime}} \pi^{E^{\prime}} d \pi_{E^{\prime}}\left\{\frac{\partial f_{M^{\prime}}}{\partial \omega^{A}} d \bar{\omega}^{M^{\prime}}+\frac{\partial f^{M}}{\partial \omega^{A}} d \bar{\pi}_{M}\right\},
\end{gathered}
$$


and we see that this last expression is symmetric in $\pi_{A^{\prime}} \ldots \pi_{D^{\prime}}$. So we have obtained that

$$
\frac{\partial}{\partial z^{A A^{\prime}}} \varphi_{B^{\prime}} \ldots D^{\prime}
$$

is symmetric in $A^{\prime}, \ldots, D^{\prime}$. This implies immediately that

$$
\varepsilon^{A^{\prime} B^{\prime}} \frac{\partial}{\partial z^{A A^{\prime}}} \varphi_{B^{\prime}} \ldots D^{\prime}=0
$$

by the skew-symmetric nature of $\varepsilon^{A^{\prime} B^{\prime}}$. We recall that

$$
\nabla^{A A^{\prime}}=\varepsilon^{B A} \varepsilon^{B^{\prime} A^{\prime}} \nabla_{B B^{\prime}}
$$

and thus

$$
\nabla^{A A^{\prime}} \varphi_{A^{\prime} \ldots D^{\prime}}=\varepsilon^{B A} \varepsilon^{B^{\prime} A^{\prime}} \nabla_{B B^{\prime}} \varphi_{A^{\prime} \ldots D^{\prime}}=0
$$

as was required.

We have to show that the integral depends only on the cohomology class defined by $f$ in $H^{0,1}\left(\mathbf{P}_{3}^{+}, H^{-n-2}\right)$. Suppose that $f=\bar{\partial} h$, where $h \in \mathcal{E}^{0}\left(\mathbf{P}_{3}^{+}\right.$, $H^{-n-2}$ ), (i.e., $h$ is represented in $\mathbf{C}^{4}-\{0\}$ by a smooth function satisfying $Y\lrcorner d h=(-n-2) h)$. Then we have

$$
\begin{aligned}
\int_{\tau^{-1}(p) \approx \mathbf{P}_{1}} \pi_{B^{\prime}} \cdots \pi_{D^{\prime}} f \wedge \theta & =\int_{\mathbf{P}_{1}} \bar{\partial}\left(\pi_{B^{\prime}} \ldots \pi_{D^{\prime}} h \wedge \theta\right) \\
& =\int_{\mathbf{P}_{1}} d\left(\pi_{B^{\prime}} \ldots \pi_{D^{\prime}} h \wedge \theta\right)=0
\end{aligned}
$$

noting that a $(2,0)$-form restricted to $\mathbf{P}_{\mathbf{1}}$ necessarily vanishes. Q.E.D.

10. Deriving the zero-rest-mass field equations from integral geometry. In the last section we showed how certain cohomology classes satisfied the zero-rest-mass field equations. We will now show how these equations can be represented in an $S U(2,2)$ invariant and coordinate free manner, and that the cohomology classes "automatically" satisfy the equations from this point of view.

We start with our basic diagram (4.4)

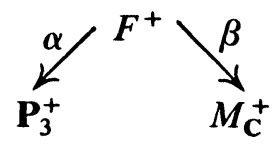

Now let $T_{\alpha}\left(F^{+}\right)$be the subbundle of the tangent bundle $T\left(F^{+}\right)$which is tangent to the fibers of the mapping $\alpha$, and let $T_{\alpha}^{*}\left(F^{+}\right)$be the dual bundle. We then have the natural projection

$$
\begin{array}{lll}
T^{*} & \stackrel{\pi_{\alpha}}{\rightarrow} T_{\alpha}^{*} \rightarrow 0 \\
\uparrow & & \uparrow \\
T & \leftarrow & \\
T & \leftarrow 0
\end{array}
$$

dual to the injection of $T_{\alpha}$ into $T$ (dropping the notational dependence on $\left.F^{+}\right)$. 
Now consider the tangential to $\alpha$ exterior differential operator

$$
d_{\alpha}=\pi_{\alpha} \circ d,
$$

where we extend $\pi_{\alpha}$ to higher order differential forms in a natural manner. In other words, we compute the exterior derivative of a differential form, and then restrict the differential form to act only on $T_{\alpha}$, the tangent vectors to the fibers of $\alpha$. We get a well-defined mapping

$$
H^{0,1}\left(F^{+}\right) \stackrel{d_{\alpha}}{\rightarrow} H^{0,1}\left(F^{+}, T_{\alpha}^{*}\right)
$$

since

$$
d_{\alpha}: \mathcal{E}^{1}\left(F^{+}\right) \rightarrow \mathcal{E}^{1}\left(F^{+}, T_{\alpha}^{*}\right),
$$

and we represent cohomology classes by $\bar{\partial}$-closed $(0,1)$-forms, as usual. The differential operator extends to differential forms with coefficients in $\alpha^{*} H^{-n-2}$ since the transition functions for $\alpha^{*} H^{-n-2}$ can be taken to be constant along the fibers of $\alpha$, and thus would be annihilated by $d_{\alpha}$. Therefore, we get a mapping

$$
H^{0,1}\left(F^{+}, \alpha^{*} H^{-n-2}\right) \stackrel{d_{\alpha}}{\rightarrow} H^{0,1}\left(F^{+}, \alpha^{*} H^{-n-2} \otimes T_{\alpha}^{*}\right) .
$$
be

One can define a vector bundle $V_{n} \rightarrow M_{\mathbf{C}}^{+}$by defining the fibers of $V_{n}$ to

$$
V_{n, x}=H^{0,1}\left(\beta^{-1}(x), \alpha^{*} H^{-n-2}\right), \quad n \geqslant 0 .
$$

This depends on the surjective mapping $\beta$, and one can verify that $V_{n}$ defined fiber-wise in this manner is indeed a holomorphic vector bundle in a natural manner (this depends on the theory of direct image sheaves [7], which we won't discuss here). There is a natural mapping

$$
\tau: H^{0,1}\left(F^{+}, \alpha^{*} H^{-n-2}\right) \rightarrow H^{0,0}\left(M_{\mathrm{C}}^{+}, V_{n}\right)
$$

obtained by restricting a $\bar{\partial}$-closed differential form representing a cohomology class in $H^{0,1}\left(F^{+}, \alpha^{*} H^{-n-2}\right)$ to a differential form on the submanifold $\beta^{-1}(x)\left(\cong \mathbf{P}_{1}(\mathbf{C})\right)$, which defines a cohomology class in $H^{0.1}\left(\beta^{-1}(x), \alpha^{*} H^{-n-2}\right)$, i.e., a point in the vector-space $V_{n}$. There is a mapping

$$
\tau_{\alpha}: H^{0,1}\left(F^{+}, \alpha^{*} H^{-n-2} \otimes T_{\alpha}^{*}\right) \rightarrow H^{0,0}\left(M_{\mathbf{C}}^{+}, V_{n}^{\alpha}\right),
$$

where $V_{n, x}^{\alpha}=H^{0,0}\left(\beta^{-1}(x), \alpha^{*} H^{-n-2} \otimes T_{\alpha}^{*}\right)$, which is defined in the same manner, and we have the following diagram:

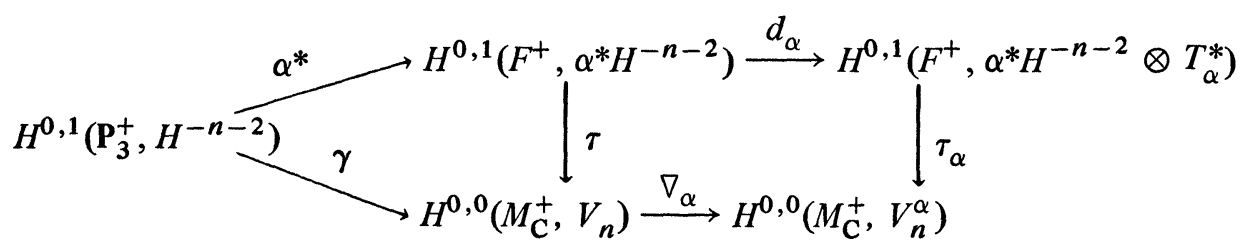

The dotted line represents the mapping induced by $d_{\alpha}$ if this is well defined.

We have the following theorem concerning this diagram. 
10.1 THEOREM. Suppose $n>0$, then

(1) $\tau$ and $\tau_{\alpha}$ are isomorphisms and $\nabla_{\alpha}$ is well defined.

(2) $V_{n, x} \cong \otimes_{\text {sym }}^{n} \mathrm{C}^{2}$.

(3) $V_{n, x}^{\alpha} \cong\left(\otimes_{\text {sym }}^{n-1} \mathbf{C}^{2}\right) \otimes \mathbf{C}^{2}$.

(4) The induced differential operator $\nabla_{\alpha}$ is the zero-rest-mass operator of spin $s=n / 2$.

(5) $\operatorname{Ker} d_{\alpha}=\operatorname{Im} \alpha^{*}$.

As a corollary of this theorem we have

10.2 Corollary. If $s>0$, then

$$
\begin{aligned}
H^{0,1}\left(\mathbf{P}_{3}^{+}, H^{-2 s-2}\right) \stackrel{\gamma}{\cong} \quad\left\{\operatorname{Ker} \nabla_{\alpha}: H^{0,0}\left(M_{\mathbf{C}}^{+}, V_{2 s}\right) \rightarrow H^{0,0}\left(M_{\mathbf{C}}^{+}, V_{2 s}^{\alpha}\right)\right\} \\
\cong \quad\{\text { self dual holomorphic solutions of the } \\
\text { zero-rest-mass equation of spin s on } \left.M_{\mathbf{C}}^{+}\right\} .
\end{aligned}
$$

In particular this corollary shows that the representation given in $\$ 9$ by an explicit integral formula is invertible.

The details of the proof of Theorem 10.1 will appear later, and we remark that all of the elements of the proof are $S U(2,2)$-invariant in nature. This implies in particular the conformal invariance of the solutions of the zerorest-mass equations, which does not follow from the integral formula given in \$9. Part (1) of Theorem 10.1 follows from the Leray spectral sequence for direct image sheaves (cf. Godement [7]), and appropriate standard cohomology vanishing theorems in several complex variables along either the fibers of $\beta$ or on $M_{C}^{+}$. Parts (2) and (3) are a computation using standard results from the theory of compact complex manifolds. Part (4) follows from an appropriate choice of basis for the vector spaces involved. That $\operatorname{Im} \alpha^{*} \subset$ $\operatorname{Ker} d_{\alpha}$ is a simple consequence of the fact that $d_{\alpha}$ corresponds to differentiation along the fibers of $\alpha$ and cohomology classes in $\operatorname{Im} \alpha^{*}$, being pullbacks along the fibers, are essentially "constant along the fibers", hence annihilated by $d_{\alpha}$. The converse statement is much deeper and involves solving appropriate $\bar{\partial} u=f$ problems locally along the fibers of $\alpha$ (the inhomogeneous Cauchy-Riemann equation for differential forms). The details will appear in a joint paper with M. Eastwood and R. Penrose, which will consider also the case $s \leqslant 0$, which is not covered by Theorem 10.1, as well as various other questions raised by the above analysis.

\section{REFERENCES}

1. A. Andreotti and F. Norguet, Problème de Levi et convexité holomorphe pour les classes de cohomologie, Ann. Sculoa Norm. Sup. Pisa 20 (1966), 197-241.

2. M. Atiyah, T. Hitchen and I. Singer, Deformations of instantons, Proc. Nat. Acad. Sci. U.S.A. 74 (1977), 2662-2663.

3. M. Atiyah and R. Ward, Instantons and algebraic geometry, Comm. Math. Phys. 55 (1977), 111-124.

4. W. Barth, Some properties of stable rank-2 vector bundles on $\mathbf{P}_{n}$, Math. Ann. 226 (1977), $125-150$.

5. S. S. Chern and J. Moser, Real hypersurfaces in complex manifolds, Acta Math. 133 (1976), 219-271.

6. G. deRham, Variétés differentiables, Hermann, Paris, 1960. 
7. R. Godement, Topologie algébrique et théorie des faisceaux, Hermann, Paris, 1964.

8. R. C. Gunning and H. Rossi, Analytic functions of several complex variables, Prentice-Hall, Englewood Cliffs, N. J., 1965.

9. R. O. Hansen and E. T. Newman, A complex Minkowski space approach to twistors, General Relativity and Gravitation 6, (1975), 361-385.

10. R. Harvey and B. Lawson, On boundaries of complex analytic varieties. I, Ann. of Math. (2) 102 (1975), 233-290; II, Ann. of Math. 106 (1977), 213-238.

11. F. Hirzebruch, Topological methods in algebraic geometry, Springer-Verlag, Berlin and New York, 1966.

12. L. Hughston, Twistor description of low-lying baryon states, Twistor Newsletter No. 1, pp. 1-6, March 1976, Oxford.

13. N. Kuiper, On conformal, flat spaces in the large, Ann. of Math. (2) 50 (1949), 916-924.

14. D. Lerner, The non-analytic version of Kerr's theorem, Twistor Newsletter No. 4, April 1977, Oxford.

15. July 1977 , Oxford.

16. James Morrow and K. Kodaira, Complex manifolds, Holt, New York, 1971.

17. R. Penrose, Twistor algebra, J. Math. Phys. 8 (1967), 345-366.

18. , The structure of space-time, Batelle Rencontres 1967, (C. M. de Witt and J. A.

Wheeler eds.), Benjamin, New York, 1968.

19. __ Twistor quantisation and curved space-time, Inter. J. Theoret. Phys. 1 (1968), 61-99.

20. __ Solutions of the zero-rest-mass equations, J. Math. Phys. 10 (1969), 38-39.

21. __ Twistor theory, its aims and achievements, Quantum Gravity: An Oxford Symposium (Isham, Penrose and Sciama eds.), Clarendon Press, Oxford, 1975.

22. __ Nonlinear gravitons and curved twistor theory, General Relativity and Gravitation 7 (1976), 31-52.

23. _ The twistor program, Reports on Math. Phys. 12 (1977), 65-76.

24. ___ Massless fields and sheaf cohomology, Twistor Newsletter No. 5, July 1977, Oxford.

25. R. Penrose and M. A. H. MacCallum, Twistor theory: an approach to the quantisation of fields and space-time, Physics Reports (Section C of Physics Letter), 6 (1972), 241-316.

26. I. Robinson, Null electromagnetic fields, J. Math. Phys. 2 (1961), $290-291$.

27. N. Tanaka, On the pseudo-conformal geometry of hypersurfaces of the space of $n$ complex variables, J. Math. Soc. Japan 14 (1962), 397-429.

28. A. Trautman, F. A. E. Pirani and H. Bondi, Lectures on general relativity, Brandeis Summer Institute in Theoretical Physics, 1964, vol. 1, Prentice-Hall, Englewood Cliffs, N. J., 1965.

29. R. Ward, The twisted photon, Twistor Newsletter No. 1, March 1976, Oxford.

30. , Curved twistor space, thesis, D. Phil., Oxford, 1977.

31. __ On self-dual gauge fields, Physics Letters, 61A (1977), 81-82.

32. R. O. Wells, Jr., Differential analysis on complex manifolds, Prentice-Hall, Englewood Cliffs, N. J., 1973.

33. R. O. Wells, Jr. and J. A. Wolf, Poincaré series and automorphic cohomology on flag domains, Ann. of Math. (2) 105 (1977), 397-448.

34. J. A. Wolf, The action of a real semisimple group on a complex flag manifold. I: Orbit structure and holomorphic arc components, Bull. Amer. Math. Soc. 75 (1969), 1121-1237; II: Unitary representations on partially holomorphic cohomology spaces, Mem. Amer. Math. Soc. no. 138, Amer. Math. Soc., Providence, R. I., 1974.

35. N. Woodhouse, Twistor cohomology without sheaves, Twistor Newsletter No. 2, June 1976, Oxford.

Department of Mathematics, Rice University, Houston, TeXas 77001 